\title{
MÁS ALLÁ DE LA DESERCIÓN: TRAYECTORIAS ACADÉMICAS EN LA EDUCACIÓN SUPERIOR EN CHILE ${ }^{1,2}$
}

\author{
Christian Blanco ${ }^{3}$ \\ Francisco Meneses ${ }^{4}$ \\ Ricardo Paredes 5
}

\section{RESUMEN}

Este estudio analiza las dinámicas de titulación, permanencia, deserción y reingreso a la educación superior en Chile, utilizando información entre los años 2007 y 2015. Se revelan características y patrones de comportamiento diferenciados según las diversas trayectorias. Se confirman los resultados de estudios previos sobre deserción y titulación, mientras que se hace una contribución inédita al explorar las trayectorias de los estudiantes que reingresan. Se presentan estadísticas descriptivas y modelos de regresiones logísticas multinomiales para evaluar la influencia de factores socioeconómicos y educativos en las diversas trayectorias en la educación superior. Se observa que cerca de la mitad de los desertores reingresa a la educación superior, mostrando importantes tasas de titulación entre estudiantes reingresantes. Además, más de la mitad de quienes reingresan se cambia de área de estudios. Estos resultados muestran que la inclusión del reingreso entre las trayectorias posibles obliga a reconsiderar la idea de que la deserción es un mero "fracaso académico". Esto exige sofisticar los marcos teóricos y empíricos, revisitar la teoría de la mismatch hypothesis y relevar la importancia de la información, de la orientación vocacional y reflexionar sobre el modo en que las políticas públicas abordan la deserción en la educación superior.

Conceptos clave: deserción, educación superior, graduación, permanencia, reingreso, titulación, trayectorias académicas.

1 Proyecto apoyado financieramente por CNED/ Convocatoria 2016.

2 Los autores agradecen especialmente a Leonor Varas, directora del DEMRE, y a Rodrigo Rolando, jefe del SIES, así como al staff profesional de ambas instituciones. También se agradece la colaboración de Paulina Berríos de la Universidad de Chile, quien asesoró a los investigadores, así como a los evaluadores, editores y correctores del CNED, cuya labor fue fundamental para la publicación de este artículo.

3 Instituto de Filosofía y Ciencias de la Complejidad, Santiago, Chile. Contacto: chblancoj@gmail.com

4 Duke University, Durham, Estados Unidos. Contacto: francisco.meneses@duke.edu

5 DUOC UC, Santiago, Chile. Contacto: rparedesm@duoc.cl 


\title{
BEYOND DROPOUTS: ACADEMIC TRAJECTORIES IN HIGHER EDUCATION IN CHILE
}

\begin{abstract}
This research addresses the dynamics of graduation, retention, desertion and readmission in higher education in Chile, from 2007 to 2015. Characteristics and behavior patterns appeared, differentiated by the distinct trajectories. This study confirms previous findings on desertion and graduation and offers the unprecedented contribution of exploring academic trajectories of readmitted students. We present descriptive statistics and multinomial logistic regression models to evaluate the influence of socioeconomic and educational factors on the diverse trajectories in higher education. We observe that about half of college dropouts re-enter the higher education system, and we note high rates of graduation among readmitted students. Moreover, over half of readmitted students change their area of study. These results indicate that the inclusion of readmittance as a possible academic trajectory, challenges the idea that desertion equates to "academic failure", and thus requires greater complexity in the theoretical and methodological frameworks on desertion, to revisit the mismatch hypothesis and an increased recognition of the importance of information, career orientation, and to reflect on how public policies address desertion in higher education.
\end{abstract}

Keyconcepts: academic trajectories, attrition, dropout, graduation, higher education, retention, persistence, readmission.

\section{Trayectorias académicas más allá de la deserción}

Aunque la deserción de la educación superior ha sido objeto de múltiples investigaciones en la última década, se le ha dado escasa atención al reingreso al sistema. Aún menos interés se observa respecto de las diferencias entre la reinserción universitaria y la del sector técnico profesional o según área de estudio. El fenómeno del reingreso al sistema obliga a matizar teórica y empíricamente el concepto de "deserción", entendido habitualmente como una forma de fracaso académico. Este estudio analiza las dinámicas de titulación, permanencia y deserción, considerando adicionalmente el reingreso a la educación superior, con el objetivo de identificar el efecto de las diversas variables que influyen en las distintas trayectorias académicas. En este estudio se observa que cerca de la mitad de los estudiantes que desertan reingresan a la educación superior, alcanzando importantes tasas de titulación. Además, se encuentra que más de la mitad de los desertores que reingresan se cambia de área de estudios. 
En las últimas décadas, Chile ha mostrado importantes avances en cobertura para todos los niveles educativos. A fines de la década de los noventa, la cobertura en educación escolar —básica y media— alcanzaba el $90 \%$ y el $60 \%$ de cobertura neta, subiendo a un $93 \%$ y un $73,6 \%$ en el año 2015. A su vez, la cobertura preescolar entre 0 a 3 años subió 23 puntos porcentuales entre 1990 y 2015, hasta alcanzar el 29,1\% en 2015 (Ministerio de Desarrollo Social, 2015). La educación superior no ha sido la excepción. Consistentemente con el aumento de egresados del nivel secundario, la cobertura bruta de educación superior pasó de 15,6\% en 1990 a un 53,1\% en 2015, en gran medida favorecido por las políticas de ayuda financiera individual —en la forma de becas y créditos- que mostraron un fuerte incremento, especialmente, desde mediados de la década del año 2000. La entrega de becas en el último quinquenio y de inversiones sobre el Crédito con Aval del Estado (CAE) parecen haber estado a la base de este crecimiento. El incremento en la demanda por educación superior ha sido absorbido en su mayoría por la oferta universitaria privada y por alternativas de educación superior técnico profesional, también privadas.

Las nuevas condiciones de la educación superior chilena y, en particular, el nuevo perfil de estudiantes, presentan importantes desafíos para las instituciones. El incremento en el acceso a la educación superior sigue estando altamente correlacionado con el ingreso (Ministerio de Desarrollo Social, 2015; SIES, 2015). Sin embargo, como nunca antes en Chile, uno de cada tres jóvenes entre 18 y 24 años del quintil más pobre accede a la educación superior (Ministerio de Desarrollo Social, 2015). Con estos avances en acceso, el foco de atención de la política y la academia nacional se ha reorientado desde la cobertura hacia la efectividad de los procesos formativos. La deserción, permanencia y la titulación oportuna son focos clave que reflejan eficiencia y calidad educativa. A su vez, dichas políticas influyen en la calidad de vida de los estudiantes y sus familias, por los efectos en los recursos destinados a la educación.

Según el Panorama de la Educación Superior en Chile del Servicio de Información de Educación Superior (SIES, 2015), el sistema pierde aproximadamente un $30 \%$ de los estudiantes que se matriculan 
el primer año, similar a lo reportado en otros países de la OCDE. También muestra que los niveles de deserción más altos están en instituciones técnicas, es decir institutos profesionales (IP) y centros de formación técnica (CFT), en carreras técnicas y en modalidades vespertinas. Los desertores son principalmente hombres mayores de 25 años, de los quintiles de ingreso más bajos, provenientes de establecimientos municipales, especialmente técnico profesionales, y que muestran bajo rendimiento académico, bajos puntajes en pruebas estandarizadas y que no cuentan con ayudas estudiantiles. Lo anterior es consistente con la literatura internacional sobre la deserción. Ante esta situación, existe una variedad de respuestas tanto institucionales como de política pública para los desafíos en permanencia y titulación de los estudiantes de educación superior, pero la gran mayoría está enfocada en los estudios universitarios, donde se sabe que la deserción es menor (por ejemplo, los propedéuticos y el Programa de Acompañamiento y Acceso Efectivo, PACE, entre otros).

La deserción global en el sistema se ha mantenido estable a través de los años (SIES, 2015). Sin embargo, mientras las universidades estatales y las privadas del Consejo de Rectores de las Universidades Chilenas (CRUCH) presentan un nivel de deserción menor al 20\%, esta alcanza el 30\% en las universidades privadas, el $36 \%$ en IP y el 37\% en CFT. No obstante, el 44\% de los desertores reingresa al sistema durante los tres años siguientes (Rolando, Salamanca, Lara \& Blanco, 2012a). Este dato enfatiza la necesidad de analizar las trayectorias académicas más allá de la deserción institucional. En efecto, mientras los estudiantes típicamente considerados "exitosos" se titulan o permanecen estudiando en el programa en el que se matricularon originalmente, se le ha dado escasa atención al fenómeno del reingreso. Reconsiderar trayectorias académicas más complejas que la tipificación binaria entre titulado y desertor es fundamental, toda vez que el proceso formativo no se acaba necesariamente en el abandono del primer programa. Por otro lado, el reingreso puede ocurrir en distintos plazos —uno, dos, tres o más años luego de la primera deserción-, y en distintas alternativas de educación superior, ya sea universitaria o técnica, en la misma o en un área del conocimiento diferente. 
El significativo volumen de estudiantes que reingresa en el corto plazo y la verificación de la existencia de diferentes alternativas de trayectorias académicas — más allá de la deserción — obligan a reconstruir tanto teórica como empíricamente el concepto de éxito y fracaso académico. Por ejemplo, un desertor de la educación superior técnico profesional que reingresa al sistema a una alternativa de formación profesional o universitaria podría ver incrementados los niveles de ingresos y empleabilidad si logra titularse y también si el reingreso es desde lo universitario hacia lo técnico profesional. El desertor que reingresa podría haber evaluado una carrera en un área distinta de la inicial, o bien, seleccionar una institución que sea de mejor calidad o que cumpla sus expectativas de modo más satisfactorio. Sin embargo, el reingreso no es garantía de éxito posterior ni certeza de permanencia y titulación del estudiante. Por ello, esta investigación exploratoria se enfoca en la primera deserción y en el primer reingreso.

El propósito más específico de este trabajo es caracterizar las dinámicas de permanencia, titulación, deserción y reingreso en la educación superior tanto universitaria como técnico profesional en Chile, identificando las variables que influyen en las decisiones de los estudiantes en cada una de las posibles trayectorias. A partir de las bases de datos del Ministerio de Educación (Mineduc) y del cuestionario de la Prueba de Selección Universitaria (PSU) del Departamento de Evaluación, Medición y Registro Educacional (DEMRE) con la cohorte que ingresó el año 2007, se generaron y analizaron estadísticas descriptivas de deserción institucional y reingreso por tipo de institución, para luego aplicar dos modelos de regresión logística multinomial, con el propósito de estudiar correlaciones condicionadas. En primer lugar, se examinaron las alternativas asociadas al estatus del estudiante luego de haberse matriculado en primer año (titulado, matriculado, desertor) y, en una segunda etapa, se evaluaron las variables asociadas al reingreso en la misma área de estudio o al reingreso en un área de estudio distinta, por tipo de institución (universitaria o técnico profesional).

Las trayectorias son abordadas a la luz de variables sociodemográficas, académicas, institucionales y geográficas. Dentro de las variables institucionales se consideraron: tipo de institución, 
área de estudio y tipo de establecimiento de enseñanza media, entre otras. Entre las académicas se tomaron en cuenta los puntajes de la Prueba de Selección Universitaria (PSU), el ranking de egreso de enseñanza media y la modalidad educativa. Dentro de las variables sociodemográficas se incluyeron el sexo, la escolaridad de la madre, el nivel de ingreso y la condición de ser beneficiario o no de ayudas estudiantiles en la forma de becas y créditos gubernamentales.

Esta aproximación innova y exige la generación de una tipología más compleja y comprensiva acerca de las trayectorias académicas. Asimismo, la investigación es relevante metodológicamente, en la medida en que aplica la tipología de trayectorias como base de análisis y comparación. Adicionalmente, constituye un aporte en términos empíricos, ya que extrae rendimientos inéditos de las bases de datos de nivel individual de la educación superior en el país, lo que es un insumo para la política pública en un segmento de la población para el cual no existen políticas específicas. Finalmente, se sostiene que esta investigación hace una contribución ética en la medida en que evita la estigmatización del desertor de primer año como un caso de fracaso académico, reconociendo su posibilidad de éxito en el reingreso en el corto plazo a la educación superior.

El estudiante que deserta y reingresa, que busca una nueva oportunidad, queda oculto tras la teoría y la investigación empírica dominante y, por ello, resulta invisible para la política pública que lo trata indiferenciadamente como cualquier estudiante. Este trabajo pretende ser un primer paso que contribuya a la resignificación del abandono de primer año — que actualmente es el indicador principal de fracaso académico- - y a la sofisticación de la mirada en torno a las trayectorias más allá de la deserción.

\section{Desarrollos teóricos acerca de las trayectorias educacionales y sus limitaciones}

Probablemente debido a la importancia de la deserción para la gestión institucional y para las políticas públicas —que requieren mecanismos efectivos y eficientes de permanencia y medición de logro académico-, la gran mayoría de las aproximaciones teóricas 
referentes a las trayectorias en la educación superior se basan en una perspectiva binaria orientada según la oposición entre éxito y fracaso académico. Como la investigación en esta materia comenzó con el objetivo de resolver problemas institucionales, tendió a utilizar información limitada y específica de cada institución. Sin embargo, análisis contemporáneos basados en estadísticas de nivel nacional permiten dar cuenta de la existencia de múltiples alternativas de trayectorias académicas, más allá de la deserción y de la graduación (SIES, 2015). La constatación de la diversidad de trayectorias, no obstante, no ha sido adecuadamente recogida hasta el momento en la literatura teórica, lo cual está exigiendo una complejización de la misma para seguir avanzando en esta línea de investigación.

El proceso de mayor interés ha sido la deserción (attrition o dropout, en la literatura anglosajona), igualada al fracaso académico y opuesta a permanencia y titulación como indicativos de éxito académico. Existen diversas acepciones y perspectivas acerca de la deserción y hay consenso en que el término dice relación con el abandono voluntario del programa académico. Generalmente, la deserción se relaciona con elementos socioeconómicos, personales, institucionales y académicos (Bordón, Canals y Rojas, 2015).

Un foco relevante del análisis de la deserción es el momento de la formación en que ocurre, donde se pueden reconocer etapas críticas (Tinto, 1987): el proceso de admisión, las primeras semanas de transición entre la educación secundaria y superior, en la última fase del primer año y antes del comienzo del segundo. Algunos autores han reconocido diferencias en el momento de la deserción -que puede ser inicial, temprana o tardía- y respecto del espacio del que se deserta, que puede ser de la institución o del sistema (González y Uribe, 2002).

La literatura distingue distintas aproximaciones para evaluar los factores que inciden en la deserción. Es posible encontrar recopilaciones de fuentes teóricas al respecto, identificando los modelos psicológicos, sociológicos y económicos que tienden a confluir en las visiones integradas más sofisticadas en la actualidad (Díaz Peralta, 2008): 
- Están los modelos psicológicos que destacan el rol de los rasgos de personalidad de los estudiantes, para quienes la actitud individual y las expectativas sociales que se tienen sobre ellos son factores fundamentales para la permanencia y abandono, un ejemplo de estos modelos es la teoría de la acción razonada (Ajzen \& Fishbein, 1977).

- Los modelos sociológicos, por otra parte, enfatizan los elementos estructurales de la sociedad. En estas perspectivas, la falta de integración social, el rendimiento académico, los niveles de satisfacción, el compromiso institucional y las condiciones socioeconómicas y socioculturales, entre otros elementos, explicarían la deserción (Spady, 1970).

- Los modelos económicos visualizan la deserción como una toma de decisión racional sobre la base de incentivos, ya sea desde los costos implicados o desde los efectos de los subsidios en la prevención de la deserción (Díaz Peralta, 2008). En los costos, se destaca el esfuerzo, la dificultad de las materias y, en particular, el costo de oportunidad. Ello explica que el centro de muchos análisis haya sido identificar el impacto que tienen los subsidios, como las becas y los créditos, en la decisión de permanecer o desertar.

La importancia de la deserción dice relación con los costos sociales que implica, lo que explicaría su predominancia en el esfuerzo científico de entender las trayectorias académicas. En primer lugar, se distinguen costos económicos, emocionales y familiares que afectarían al estudiante (Tinto, 1987). A su vez, habría costos para las instituciones, las que verían reducidos sus ingresos y disminuirían sus indicadores institucionales de éxito. También habría efectos sistémicos respecto de la credibilidad del sistema en su conjunto, en tanto la deserción afecta la reputación de las instituciones y tiene efectos monetarios (Tracey \& Florian, 2016).

Probablemente el modelo que provee una visión más integrada —y por ello es más frecuentemente utilizado en la investigación sobre deserción- es el modelo Student Integration Model, conocido bajo la sigla SIM (Tinto, 1975). En su base está la idea de que niveles deficitarios de integración estudiantil en las dimensiones académica 
y social definen las decisiones de los estudiantes de permanecer o abandonar. Las decisiones de deserción se relacionan con la evolución de elementos tanto del agente como de la estructura institucional, incluyendo los niveles de compromiso con las metas personales y de compromiso institucional. Estos procesos tienen a su vez como entradas las calificaciones previas, los atributos individuales y familiares, así como las condiciones contextuales pedagógicas y sociales. Algunos estudios indican que, aunque la integración social y académica son relevantes a la hora de predecir la persistencia, esos factores varían en importancia para todos los estudiantes (Díaz, 2008).

Existen aproximaciones más modernas que ponen en valor ideas como la orientación vocacional y la información para la toma de decisiones. Algunas de las visiones más complejas —aunque herederas de la tradición de Tinto- se centran en el ajuste entre el estudiante, la institución y el programa de estudios, considerando tanto factores económicos, psicológicos y sociales. La mismatch hypothesis (Sander, 2004) evalúa si el estudiante y la institución se ajustan y son un buen complemento, analizando la posibilidad de que los estudiantes estuviesen mejor en otra institución o programa. En esta línea, algunas investigaciones realizan un análisis de la literatura sobre las políticas de acción afirmativa en EE.UU., mostrando que en ciertos casos tales políticas podrían beneficiar o dañar a los estudiantes, según su nivel de mismatch (Arcidiacono \& Lovenheim, 2016).

Solo unos pocos ejemplos de aproximaciones a estos procesos han incorporado trayectorias académicas más allá de la deserción. Para analizar la deserción más allá del efecto binario, algunos autores norteamericanos proponen centrarse en la retención, deserción y reingreso, en el corto plazo, de los estudiantes a la educación superior, de forma diferenciada (Stratton, O'Toole $\&$ Wetzel, 2008). Estos investigadores conceptualizan el proceso de deserción y reingreso en el plazo de un año como stopout, es decir, como aquellos estudiantes que suspenden sus estudios y vuelven a ingresar posteriormente en el corto plazo: 
... reconocemos que muchos estudiantes que parecen abandonar sus estudios cuando se usa una medición en el tiempo de abandono, como es no aparición en el segundo año, realmente reingresan pronto y continúan sus estudios superiores. Esos no son desertores (dropouts) sino que stopouts (Stratton et al., 2008).

Esta situación —que se iniciaría con las limitaciones de datos- obliga a introducir mayor complejidad a la teoría acerca de las trayectorias académicas en la educación superior.

Sin embargo, el concepto de stopout es más aplicable en un contexto como el estadounidense que está basado en el sistema de college, donde el currículo es más flexible y los estudiantes cursan materias de áreas más diversas. En Chile, al retornar a la educación superior en el plazo de un año es más probable que el estudiante deba comenzar un programa. En el caso del sistema chileno, al tener los programas currículos más rígidos, es menos correcto referirse a ellos como stopout.

En Chile, el primer trabajo que conceptualiza el reingreso se realizó en el Sistema de Información de Educación Superior:

... desertar de una carrera de Educación Superior en un determinado año no implica necesariamente una deserción definitiva. De hecho, puede ser que el estudiante que deserta solo haya suspendido sus estudios en forma temporal debido a diversos motivos (salud, vocación, familiar o económico) o haya reingresado a un programa de otra institución (Rolando, Salamanca, Lara y Blanco, 2012, ).

En ese trabajo —-más bien por disponibilidad de datos que por motivos teóricos- se conceptualizó el reingreso como "reingreso en el corto plazo", es decir, se consideraron tres años después de la deserción de primer año. El estudio del SIES se centra en las diferencias por tipo de institución, tipo de carrera y establecimiento de origen, vinculando el reingreso con condiciones socioeconómicas. Sin embargo, no profundiza en las trayectorias de los estudiantes reingresados. 
La aproximación más correcta requiere de una teoría de las trayectorias que supere el reduccionismo binario basado en el éxito y el fracaso académico. Más allá de la deserción, el estudiante que no se gradúa ni permanece en su institución y programa de estudio puede desertar definitivamente del sistema, o bien, reingresar a distintas alternativas de educación superior. Por lo anterior, nos referiremos a reingreso cuando el estudiante se retira de su programa para volver en el futuro no limitado al plazo de un año, sino que considera todos los años disponibles. Otros estudios para Chile avanzan en esta misma dirección, incluyendo el concepto de "transferencia de institución" para entender las trayectorias académicas (Santelices, Catalán, Kruger \& Horn, 2016).

\section{Revisión de la literatura empírica}

Como consecuencia del predominio de aproximaciones teóricas basadas en la oposición entre éxito y fracaso académico, la mayoría de los estudios empíricos busca encontrar aquellos factores que influyen en mayor medida en la deserción para — eventualmente- ofrecer alternativas de intervención basadas en medidas preventivas que favorezcan la permanencia y titulación.

La literatura internacional y nacional en torno a la deserción en la educación superior confluye en la idea general de que se trata de un proceso multidimensional y multifactorial, donde son importantes los elementos de carácter individual y colectivo, así como los elementos personales e institucionales y académicos y económicos, entre otros (Bordón et al., 2015; Calcagno, Bailey, Jenkins, Kienzl \& Leinbach, 2008; Goldrick-Rab, 2010; Melguizo, Sanchez \& Velasco, 2016; Melguizo, Torres \& Jaime, 2011; Yorke \& Longden, 2004).

En Chile se ha demostrado —usando regresiones logísticasque los mejores resultados en la Prueba de Selección Universitaria (PSU), las más altas notas de enseñanza media y el mejor ranking de egreso tienen una correlación con la retención de estudiantes y que existe un efecto positivo adicional en estudiantes que son beneficiados por becas y créditos (Blanco \& Meneses, 2013; Bordón et al., 2015; Contreras, Gallegos, \& Meneses, 2009). Otros autores 
han investigado las trayectorias educacionales y laborales de los estudiantes de la educación escolar técnico profesional, encontrando que provenir de un colegio técnico profesional en la secundaria, tener bajo nivel socioeconómico y ser de género masculino incrementan la probabilidad de desertar en la educación superior (Larrañaga, Cabezas y Dussaillant, 2014).

Existen diversos ejemplos de políticas para disminuir la deserción en la educación superior. Algunos investigadores se han enfocado en políticas de nivelación (Calcagno et al., 2008) y apoyo psicosocial (Bailey, Jeong \& Cho, 2010). Existe evidencia diversa para el caso de Chile sobre el impacto de las políticas remediales en la educación universitaria (Catrileo, Lobos y Sereño, 1991; Devés \& Mora-Curriao, 2014; Gallardo, Lorca, Morrás y Vergara, 2014).

Los estudios para Chile acerca de permanencia y titulación de los estudiantes en la educación superior —que incluyen tanto la formación técnico profesional como universitaria de formacomparada son pocos. En un trabajos anteriores analizaron los efectos de las becas y créditos para predecir el ingreso de los estudiantes a la educación superior, mostrando el impacto diferenciado de becas y créditos en la formación superior técnico profesional y universitaria (Blanco y Meneses, 2013). Otros esfuerzos, que utilizan la prueba Simce como variable de control académico y métodos de matching, concluyeron que los estudiantes provenientes de una modalidad técnico profesional de nivel secundario, que hayan continuado su formación en la educación superior en la misma área de estudios, tienen menor probabilidad de desertar (Farías \& Sevilla, 2015). La presente investigación busca favorecer una aproximación sistémica a la educación superior, incluyendo su vertiente universitaria y técnico profesional.

En términos empíricos, el primer esfuerzo en el estudio del fenómeno del reingreso fue del SIES en el año 2012 (Rolando et al., 2012a), que estimó que un 44\% de los estudiantes que desertan en primer año vuelven a ingresar dentro de los siguientes tres años a la educación superior. Además, se encontraron importantes diferencias según tipo de carrera, tipo de institución y tipo de establecimiento 
de enseñanza media, vinculando un mayor porcentaje de reingreso con niveles socioeconómicos más altos. En años sucesivos, ha habido esfuerzos que han abordado los determinantes de la persistencia en la educación superior chilena y que incluyen el reingreso a la misma. Mediante una base de cuatro cohortes, regresiones logísticas y una metodología de matching, se ha encontrado que la permanencia en la educación superior y el reingreso a la misma se asocia a mejores condiciones socioeducativas y financieras (Santelices et al., 2016). En la presente investigación se busca complementar estos esfuerzos, revisando qué ocurrió con aquellos estudiantes que reingresaron, si se titularon, permanecieron o desertaron definitivamente.

Salvo las citadas excepciones, la literatura en torno a las trayectorias académicas en el mundo, en América Latina y en Chile - en general - analiza la continuidad de los estudiantes en la educación superior mediante estadísticas descriptivas y regresiones logísticas donde la variable dependiente es binaria (deserción versus permanencia). Si bien ha habido importantes contribuciones en esta línea tradicional que reduce las trayectorias al éxito y el fracaso académico, y aunque existen buenas razones para pensar que las variables asociadas a la deserción pueden estar también vinculadas al reingreso, se considera necesario avanzar hacia estudios empíricos que asuman la complejidad teórica y apliquen herramientas metodológicas más sofisticadas. Es importante reconocer que las variables que definirían la deserción afectan de manera distinta al reingreso y que otras variables pueden también estar influyendo. Tratar a ambos grupos sin distinción podría generar resultados sesgados por deficiencias de especificación y conducir a recomendaciones de política pública limitadas o definitivamente equivocadas.

\section{Categorización de trayectorias, metodología y datos}

Debido a que la literatura tradicional entiende habitualmente a los estudiantes que no se han graduado como matriculados o bien como desertores, la primera actividad teórico-metodológica es identificar y tipificar las trayectorias de los estudiantes en educación superior. Siguiendo procedimientos registrados en la literatura (Stratton et 
al., 2008), se identificaron primeramente las posibles trayectorias educacionales, para luego describir sus perfiles estadísticos y buscar un modelo econométrico que permita analizarlas, distinguiendo en este caso entre educación universitaria y educación técnico profesional, además de área de estudio. Emergen de esta reflexión siete posibles trayectorias luego del ingreso a la educación superior:

a. Continuación hasta titularse. Este tipo ideal de trayectoria corresponde al caso de éxito más tradicional.

b. Permanencia en la carrera de origen, que corresponde a aquellos estudiantes que siguen su programa regularmente y no se ha titulado. Esto es normal, ya que la titulación tiende a demorar en promedio un 30\% más de la duración formal (SIES, 2015).

c. Reingreso a la misma área de estudios y al mismo tipo de institución después de desertar de la carrera de ingreso original. Esta es la primera categoría que se asocia con la reconsideración de la deserción, entendiendo que la salida es una suspensión temporal de los estudios (stopout) o una transferencia de institución, manteniendo el tipo de institución (técnico profesional o universitaria).

d. Reingreso a otra área de estudios después de desertar de la carrera de ingreso original, manteniendo el mismo tipo de institución.

e. Reingreso a la misma área de estudios después de desertar de la carrera original, pero a un tipo de institución distinta.

f. Reingreso a un tipo de institución distinta y a un área de estudios distinta.

g. Deserción del sistema sin reingreso. Se considera deserción "de mediano plazo", solo por la disponibilidad de datos, ya que es posible que el desertor vuelva a ingresar a la educación superior una década después. 


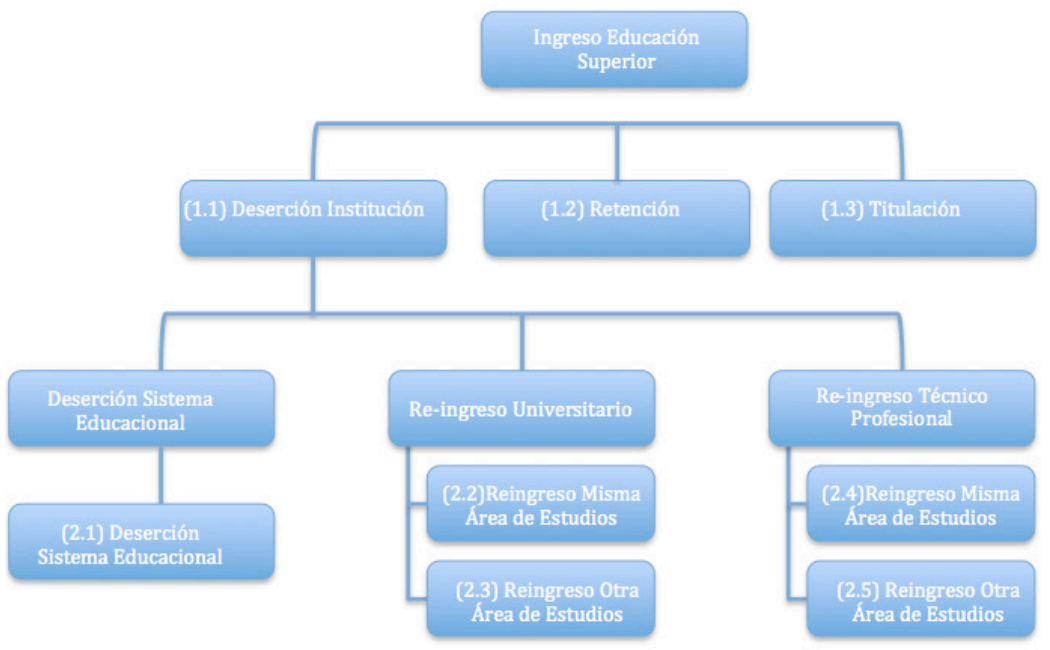

Figura 1. Diagrama de flujos de trayectorias de educación superior, incluyendo ingreso, deserción, permanencia, titulación y alternativas de reingreso.

Fuente: Elaboración propia.

La secuencia de elecciones representada en la Figura 1 procuró analizar a través de correlaciones condicionadas usando regresiones en dos etapas. En la primera, mediante multinomial logits (Mlogit), se analizó el primer set de posibilidades, esto es, estudiantes que lograban: (1.1) titularse; (1.2) permanecer matriculado; (1.3) no mantenerse y desertar de su institución. Esta es la fase confirmatoria, en la medida en que se esperaba corroborar resultados previos y aportar al cúmulo de literatura nacional e internacional sobre determinantes de la graduación, permanencia y deserción, utilizando las metodologías típicas. En una segunda etapa, se desagregaron los estudiantes que desertaron de sus instituciones y se utilizaron multinomial logits para predecir si el estudiante reingresaba: (2.1) al sector técnico profesional a la misma área de estudios; (2.2) al sector técnico profesional a otra área de estudios; (2.3) al sector universitario a la misma área de estudios; (2.4) al sector universitario a otra área de estudios; o bien, (2.5) desertaba definitivamente del sistema de educación superior.

Se distinguió a los estudiantes que ingresaron a universidades de aquellos que lo hicieron en instituciones de carácter técnico 
profesional. Se incluyeron variables independientes tradicionalmente utilizadas en la literatura en las dimensiones personal, académica, institucional y sociodemográfica (ver Anexo, Figura 1).

En la dimensión académica, las variables utilizadas fueron las notas de enseñanza media (NEM), la PSU y el ranking de enseñanza media. En la dimensión institucional, las variables fueron modalidad de enseñanza media (técnico profesional o científico humanista), dependencia del establecimiento de enseñanza media, institución (CFT, IP o universidad) y área de estudio.

En la dimensión socioeconómica y demográfica, las variables consideradas fueron el género, el nivel educacional de la madre, el nivel de ingreso familiar y si cuenta con beneficios estatales para la educación superior. Aunque otras variables como el avance curricular, la proporción de cursos aprobados respecto de los cursos inscritos, las calificaciones de la educación superior, entre otras, serían muy relevantes de incorporar, no se dispone de datos a nivel nacional de los resultados académicos de la educación superior. Por ello, y siguiendo la literatura, se consideraron las variables académicas asociadas a la admisión: el ranking de educación media del estudiante respecto de su generación en su establecimiento y el resultado obtenido por el estudiante en las pruebas estandarizadas de selección universitaria (PSU), en los ámbitos de lenguaje y matemática.

Para la dimensión institucional se incluyeron variables del establecimiento de enseñanza media (modalidad científico humanista o técnico profesional, y dependencia, indicativa del nivel socioeconómico de los estudiantes), y variables de la institución de educación superior (universidad, instituto profesional y centro de formación técnica, y área del conocimiento del programa en que el estudiante está matriculado).

Además de la información del SIES, se incluyeron variables de la encuesta que el DEMRE aplica al momento de inscribir en la PSU. Se utilizaron los años de escolaridad de la madre, altamente correlacionados con el nivel socioeconómico, y el ingreso mensual familiar declarado por el estudiante, así como la condición de beneficiario de ayuda financiera y el género. 
Por último, el estado de matrícula, deserción, titulación o reingreso se recogió en la variable de trayectoria, que opera como la variable dependiente del estudio.

\section{Resultados descriptivos}

\subsection{Permanencia, deserción y titulación}

De los más de 238 mil graduados de enseñanza media del año 2006, en 2007 cerca de 32 mil ingresaron a las universidades del CRUCH; 26 mil a universidades privadas; y 30 mil a la educación superior técnico profesional, integrada por las llamadas instituciones vocacionales. De quienes optaron por instituciones vocacionales, más de 11 mil ingresaron a un centro de formación técnica (CFT), que entregan formación técnica de dos años y 19 mil a institutos profesionales (IP), que además de carreras técnicas, ofrecen carreras profesionales de cuatro años. Ello totaliza cerca de 88 mil estudiantes en las 193 instituciones de educación superior para el año de referencia (Anexo Tabla 1D).

No todos los graduados de la educación secundaria ingresan a la educación superior habiendo rendido la PSU. Mientras el 99\% de los estudiantes que entró a una universidad del CRUCH rindió la PSU, este porcentaje baja a $97 \%$ en las universidades privadas. De la totalidad de estudiantes que ingresó a la educación técnico profesional, poco más de 24 mil rindieron la PSU (8 mil a un CFT y 16 mil a un IP). En total, un $81 \%$ de los estudiantes que egresaron de cuarto medio en 2006 y que ingresaron inmediatamente a la educación superior técnico profesional rindió la PSU. Solo se consideraron en este estudio aquellos estudiantes que rindieron la PSU, lo que permite contar con información académica y socioeconómica para el 92\% del total de estudiantes que hicieron ingreso inmediato a la educación superior luego de egresar de la enseñanza media (ver Anexo, Tabla 1D).

En la Tabla 1 se observa que de los 88 mil estudiantes de la cohorte 2007, al segundo año, un 76\% sigue estudiando en la misma institución, en cambio, un 15\% ha desertado del sistema y un 10\% ha reingresado. Al segundo año la tasa de deserción y reingreso se 
igualan en un $18 \%$. Otros estudios considerarían que la deserción seria del 36\%, en al tanto medir el reingreso, la deserción disminuye a la mitad en el tercer año. Al séptimo año un 32\% continuaba estudiando ( $15 \%$ de matriculados y $17 \%$ de reingresantes), un $42 \%$ se había titulado y un $25 \%$ había desertado del sistema. En tanto, al noveno año, solo un $26 \%$ de los estudiantes ha desertado, en tanto un $60 \%$ se ha titulado, un 4\% sigue en la institución donde comenzó y un $11 \%$ esta estudiando en otra institución.

Tabla 1

Porcentaje y número de estudiantes por año y condición de matrícula, titulación y deserción de los egresados de educación secundaria en 2006 que ingresaron a la educación superior en 2007

\begin{tabular}{cccccccccc}
\hline \multicolumn{4}{c}{ Educación superior (Porcentaje) } & \multicolumn{4}{c}{ Educación superior (Número) } \\
\hline Año & Matrícula & Deserción Reingreso & Titulación & Matrícula & Deserción & Reingreso Titulación \\
\hline 1 & $100 \%$ & $0 \%$ & $0 \%$ & $0 \%$ & 88.401 & - & - & - \\
2 & $76 \%$ & $15 \%$ & $10 \%$ & $0 \%$ & 66.797 & 13.021 & 8.583 & - \\
3 & $59 \%$ & $18 \%$ & $18 \%$ & $4 \%$ & 52.447 & 16.354 & 15.740 & 3.860 \\
4 & $50 \%$ & $20 \%$ & $21 \%$ & $8 \%$ & 44.444 & 17.796 & 18.766 & 7.395 \\
5 & $40 \%$ & $21 \%$ & $22 \%$ & $17 \%$ & 35.099 & 18.846 & 19.255 & 15.201 \\
6 & $26 \%$ & $24 \%$ & $20 \%$ & $29 \%$ & 23.325 & 21.436 & 17.640 & 26.000 \\
7 & $15 \%$ & $25 \%$ & $17 \%$ & $42 \%$ & 13.031 & 22.348 & 15.455 & 37.567 \\
8 & $8 \%$ & $26 \%$ & $14 \%$ & $53 \%$ & 6.769 & 22.666 & 12.386 & 46.580 \\
9 & $4 \%$ & $26 \%$ & $11 \%$ & $60 \%$ & 3.526 & 22.609 & 9.390 & 52.876 \\
\hline
\end{tabular}

Fuente: Elaboración propia sobre la base de Mineduc 2006 y SIES (2007 - 2015).

Aunque estas cifras tienden a lo reportado en la literatura nacional e internacional, existen leves diferencias entre algunas cifras del SIES y las de otros autores que analizan las tasas de deserción de la misma cohorte, sin diferenciar el año de egreso de la enseñanza media o si rindieron la PSU (Bordón et al., 2015). En este trabajo se estima que la deserción llegaría al 31,8\%, lo que es mayor al $25 \%$ ( $15 \%$ deserción más $10 \%$ de reingreso a primer año) aquí reportado. La diferencia puede obedecer a que el presente estudio considera exclusivamente a los estudiantes recién egresados de la educación secundaria (denominado ingreso inmediato) y no a todos los matriculados de primer año, que pueden provenir de generaciones anteriores. 
La inspección visual de la Figura 2 revela importantes diferencias entre los estudios técnico profesionales y los universitarios. El porcentaje de desertores es muy alto el primer año, especialmente en el sector técnico, CFTs e IPs, para luego ir estabilizándose en los años siguientes sobre el 30\%. Esta cifra alcanza un 40\% en estudios técnico profesionales y un $20 \%$ en estudios universitarios, aproximadamente, en concordancia con la literatura.

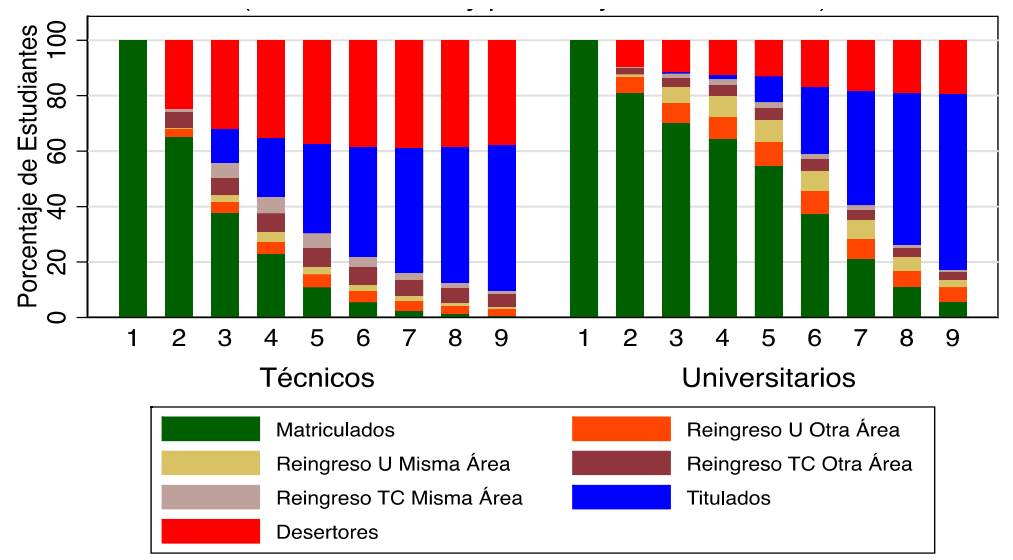

Figura 2. Porcentaje de estudiantes técnicos y universitarios por año según condición de matrícula, titulación, deserción y reingreso de los graduados de educación secundaria en 2006 que ingresaron a la educación superior en 2007. El nivel técnico incluye profesionales no universitarios de estas instituciones.

Fuente: Elaboración propia sobre la base de Mineduc 2006 y SIES (2007 - 2015).

Al segundo año, la deserción sigue siendo mucho más alta en el sector técnico profesional (25\%) que en el universitario (10\%), mientras que la tasa de reingreso es la misma (10\%) para ambos tipos de instituciones. Las instituciones técnico profesionales presentan tasas de titulación algo más altas al séptimo año (45\% técnicos versus $41 \%$ universitario). Esto se explicaría en gran medida por el mayor tiempo de duración de las carreras universitarias. Al noveno año la tendencia se revierte: la titulación en el sector técnico profesional alcanza un 53\% y en el sector universitario llega al 64\% (valores absolutos presentados en la Tabla 1E en el Anexo). 


\subsection{Patrón de reingreso}

Los resultados presentados revelan por primera vez el panorama de la permanencia, deserción y titulación al noveno año, el cual sigue la tendencia identificada por estudios previos de menor alcance. Sin embargo, en la literatura nacional e internacional los esfuerzos por abordar la complejidad del reingreso son casi inexistentes, más allá de la estimación del 44\% de reingreso en los siguientes tres años estimado por el SIES. Con el presente estudio es posible replicar tal cálculo, encontrando que un $49 \%$ de los estudiantes que se retira de su programa original reingresa en el tercer año. Esta leve diferencia se explica porque la base de cálculo del SIES considera a la totalidad de los estudiantes, a diferencia de este estudio que incluye a aquellos de primer año que rindieron PSU para ingresar.

Ya en segundo año comienza el proceso de reingreso con 10\%, que puede corresponder al mismo o a distinto tipo de institución, así como a la misma área de estudios o a una diferente. Para ambos tipos predomina levemente el reingreso al mismo tipo de institución, lo que es esperable ya que se mantiene el perfil (71\% para el sector universitario y $66 \%$ para el sector técnico).

En la Tabla 2 se muestra el porcentaje de estudiantes que se cambia de área de estudio. Para los estudiantes que desertan el primer año del sector universitario, un $46 \%$ de los que reingresa al sector universitario, se cambia de área de estudios. En tanto, para los estudiantes del sector técnico que reingresan, un 39\% de los que vuelve al sector técnico, se cambia de área de estudio al segundo año. La Tabla 2 muestra que los reingresantes en gran medida tienden a cambiar de área de estudio, lo que sugiere que el factor vocacional, de información y de orientación serían elementos importantes al momento de la deserción y la decisión de reingreso. Para la mayoría de los años, más de la mitad de los reingresantes lo hace en un área distinta a la inicial. El patrón de cambio de área va modificándose a medida que el reingreso es más tardío. Como se observa en la Tabla 2, el cambio de área es más frecuente después del segundo año, para todas las combinaciones. Si el reingreso es inmediato, es más probable que se mantenga el área de estudios. 
Dos mecanismos derivados de la matching hypothesis parecen estar asociados con este fenómeno: el estudiante ingresó a un área que no era de su interés inicial o bien el área de su interés no fue buen match para él una vez experimentado el primer año. El cambio de área es más prevalente en aquellos estudiantes que se matricularon inicialmente en una universidad. Los motivos de estas diferencias demandan estudios más profundos que analicen los perfiles de los reingresantes, para identificar las razones del mismatch.

Tabla 2

Porcentaje de estudiantes reingresantes a la educación superior que se cambian de área de estudio por tipo de institución y año de reingreso

\begin{tabular}{ccccc}
\hline \multirow{2}{*}{ Año } & \multicolumn{2}{c}{ Desertor universitario (\%) } & \multicolumn{2}{c}{ Desertor técnico (\%) } \\
\cline { 2 - 5 } & $\begin{array}{c}\text { Reingresante } \\
\text { técnico (\%) }\end{array}$ & $\begin{array}{c}\text { Reingresante } \\
\text { universitario (\%) }\end{array}$ & $\begin{array}{c}\text { Reingresante } \\
\text { técnico (\%) }\end{array}$ & $\begin{array}{c}\text { Reingresante } \\
\text { universitario (\%) }\end{array}$ \\
\hline 2 & $47 \%$ & $46 \%$ & $39 \%$ & $49 \%$ \\
3 & $65 \%$ & $55 \%$ & $54 \%$ & $62 \%$ \\
4 & $65 \%$ & $53 \%$ & $52 \%$ & $56 \%$ \\
5 & $69 \%$ & $53 \%$ & $58 \%$ & $62 \%$ \\
6 & $69 \%$ & $54 \%$ & $63 \%$ & $66 \%$ \\
7 & $74 \%$ & $53 \%$ & $70 \%$ & $68 \%$ \\
8 & $76 \%$ & $55 \%$ & $74 \%$ & $72 \%$ \\
9 & $82 \%$ & $66 \%$ & $85 \%$ & $77 \%$ \\
\hline
\end{tabular}

Fuente: Elaboración propia sobre la base de Mineduc 2006 y SIES (2007 - 2015).

En la Tabla 3 se describen las secuencias origen y destino de las áreas de estudio de los estudiantes reingresantes, entre primer y cuarto año. Las carreras en las áreas de agricultura, ciencias y servicios tienen una retención por área del conocimiento menor al $40 \%$, mientras que las ciencias sociales retienen sobre el $60 \%$ de los desertores. Es necesario profundizar en los elementos asociados a la orientación vocacional por área de estudio, ya que los patrones de matching son claramente diferenciados. 
Tabla 3

Porcentaje de estudiantes reingresantes a la educación superior según concordancia de áreas de origen y destino

\begin{tabular}{lcccccccc}
\hline & AG & CI & CS & ED & HA & II & SS & SE \\
\hline Agricultura (AG) & $35 \%$ & $4 \%$ & $12 \%$ & $6 \%$ & $7 \%$ & $10 \%$ & $16 \%$ & $10 \%$ \\
\hline Ciencias (CI) & $2 \%$ & $34 \%$ & $14 \%$ & $6 \%$ & $4 \%$ & $18 \%$ & $15 \%$ & $7 \%$ \\
\hline Ciencias sociales (CS) & $1 \%$ & $3 \%$ & $61 \%$ & $9 \%$ & $6 \%$ & $5 \%$ & $9 \%$ & $6 \%$ \\
\hline Educación (ED) & $1 \%$ & $3 \%$ & $17 \%$ & $44 \%$ & $8 \%$ & $5 \%$ & $13 \%$ & $9 \%$ \\
\hline Humanidades y Artes (HA) & $1 \%$ & $4 \%$ & $20 \%$ & $14 \%$ & $41 \%$ & $5 \%$ & $7 \%$ & $8 \%$ \\
\hline Ingeniería e industrias (II) & $3 \%$ & $6 \%$ & $14 \%$ & $5 \%$ & $5 \%$ & $51 \%$ & $8 \%$ & $7 \%$ \\
\hline $\begin{array}{l}\text { Salud y servicios de salud } \\
\text { (SS) }\end{array}$ & $1 \%$ & $2 \%$ & $14 \%$ & $10 \%$ & $3 \%$ & $5 \%$ & $60 \%$ & $6 \%$ \\
\hline Servicios (SE) & $2 \%$ & $4 \%$ & $20 \%$ & $12 \%$ & $5 \%$ & $7 \%$ & $19 \%$ & $32 \%$ \\
\hline
\end{tabular}

Nota: cifras en negritas muestran reingreso a la misma área del conocimiento. Origen (vertical) y destino(horizontal).

Fuente: Elaboración propia sobre la base de Mineduc 2006 y SIES (2007 - 2015).

Además, es necesario explorar las path dependencies que se observan según área de estudio, en la medida en que habría una cierta afinidad electiva de la primera área respecto de la segunda en algunos casos, mientras que en otros es menos claro. Por ejemplo, quienes desertan de artes y humanidades reingresan en mayor proporción a ciencias sociales (pero no a la inversa), mientras que quienes desertan de ciencias suelen reingresar a ingenierías en una mayor proporción (y viceversa). Cabe señalar que estas y otras relaciones encontradas, evidentemente, son de asociación y se requeriría mayor profundidad en el análisis para establecer vínculos de causalidad.

\subsection{Patrón de comportamiento de estudiantes de instituciones técnico profesionales}

A continuación, en la Figura 3 se distinguen los estudiantes del sector técnico profesional según pertenezcan a un según CFT o IP. La inspección visual revela patrones similares a los encontrados previamente, pero las tasas de titulación se incrementan antes en los CFT por la menor duración promedio de las carreras. 


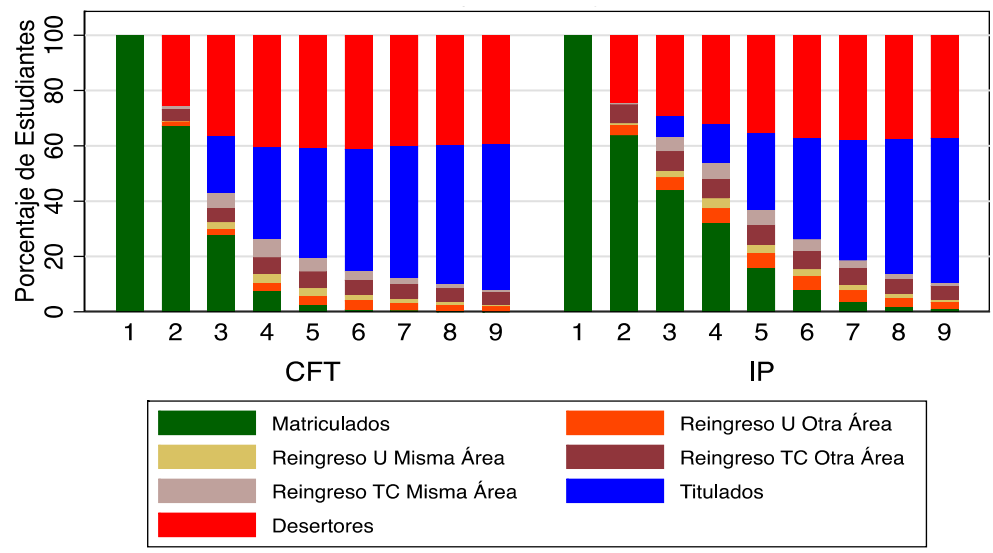

Figura 3. Porcentaje de estudiantes de CFT e IP por año según condición de matrícula, titulación, deserción y reingreso de los graduados de educación secundaria en 2006 que ingresaron a la educación superior en 2007.

Fuente: Elaboración propia sobre la base de Mineduc 2006 y SIES (2007 - 2015).

En la Tabla 4 se presenta un perfil estadístico de los estudiantes según las distintas trayectorias. En línea con la literatura nacional, los desertores tienen bajo ranking, baja PSU, bajos ingresos, vienen de establecimientos municipales de modalidad TP, son hombres y no cuentan con ayudas estudiantiles. Si bien los titulados de instituciones técnico profesionales tienen mejor ranking y cuentan con más ayudas estudiantiles, no tienen mejor PSU ni más ingresos que los reingresantes universitarios. Quienes se titulan de una institución técnico profesional tienden a ser estudiantes de mayor ranking escolar, pero no necesariamente de un nivel socioeconómico mayor o mayor puntaje PSU. Sin embargo, el perfil del reingresante universitario sí se asocia a un mayor nivel socioeconómico y a una mejor PSU, lo que lo hace similar al perfil de quienes acceden a la universidad el primer año, lo que podría explorarse desde la perspectiva teórica del mismatch institucional. 
160 MÁS ALLÁ DE LA DESERCIÓN: TRAYECTORIAS ACADÉMICAS EN LA EDUCACIÓN SUPERIOR EN CHILE - Ch. Blanco, F. Meneses y R. Paredes

Tabla 4

Perfil estadístico de estudiantes técnico profesionales de los graduados de educación secundaria en 2006 que ingresaron a la educación superior en 2007 al cuarto año, según condición de deserción, permanencia y reingreso técnico o universitario

\begin{tabular}{lccccc}
\hline & Desertor & Matriculado & $\begin{array}{c}\text { Reingresante Reingresante } \\
\text { técnico }\end{array}$ & $\begin{array}{c}\text { Titulado } \\
\text { universitario }\end{array}$ & \\
\hline Ranking escolar & $43 \%$ & $49 \%$ & $42 \%$ & $47 \%$ & $57 \%$ \\
PSU promedio & 414 & 461 & 424 & 467 & 444 \\
Modalidad TP & $37 \%$ & $29 \%$ & $32 \%$ & $24 \%$ & $30 \%$ \\
P. Pagado & $2 \%$ & $7 \%$ & $4 \%$ & $8 \%$ & $2 \%$ \\
P. Subvencionado & $31 \%$ & $48 \%$ & $42 \%$ & $46 \%$ & $39 \%$ \\
Escolaridad madre & 10,2 & 11,2 & 11,0 & 11,7 & 10,1 \\
Ingreso familiar en US\$ & 536 & 750 & 667 & 830 & 517 \\
Mujer & $46 \%$ & $46 \%$ & $46 \%$ & $54 \%$ & $65 \%$ \\
Ayuda estudiantil & $23 \%$ & $40 \%$ & $24 \%$ & $33 \%$ & $48 \%$ \\
\hline
\end{tabular}

Fuente: Elaboración propia sobre la base de Mineduc 2006 y SIES (2007 - 2015).

En el Anexo pueden encontrarse los perfiles en años sucesivos. Se destaca que, entre el cuarto, séptimo y noveno año caen en importancia las ayudas estudiantiles para la titulación, lo que implica que en el largo plazo las dificultades económicas tienden a ser una barrera más fácil de superar, probablemente porque muchos estudiantes ya trabajan. Además, las mujeres tienden a representar un porcentaje mayor de los titulados en los primeros años, lo que favorece a los hombres en años posteriores.

En síntesis, mejores condiciones académicas y socioeconómicas se asocian a una menor probabilidad de deserción. En el caso de los reingresantes, aquellos que lo hacen al sector universitario presentan indicadores más altos que los estudiantes que se mantienen en el sector técnico profesional o que reingresan a este sector. Estos resultados son relevantes no solo teóricamente, sino también en términos de políticas públicas, especialmente en contextos de ampliación del beneficio de gratuidad a CFT e IP.

\subsection{Patrón de comportamiento de estudiantes de instituciones universitarias}

Para los estudiantes universitarios, si bien se observa un patrón similar en términos de reducción progresiva de los matriculados a medida que pasan los años, la cantidad de estudiantes que deserta 
del sistema es menor. Después de nueve años, para las universidades del CRUCH, menos del 18\% de los estudiantes deserta, mientras que esta cifra alcanza el 22\% en universidades privadas. Sobre el 64\% de los estudiantes se titula después de nueve años en el sector universitario y más de un 17\% continúa estudiando, lo que significa que sobre un $80 \%$ los estudiantes no han desertado después de nueve años (Figura 4, Anexo Tabla 1E 2). El sistema, por tanto, aparenta ser muy poco eficiente en términos de titulación, pero no necesariamente en términos de deserción. Esto se debe en parte al proceso de reingreso (entre un $10 \%$ y un 20\% cada año), sumado a la larga duración de las carreras y los atrasos en la graduación.

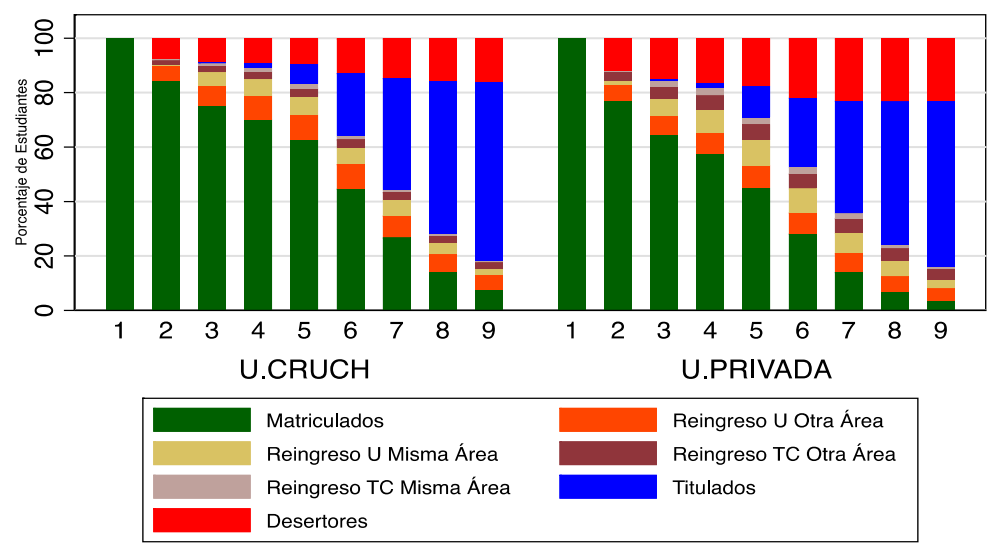

Figura 4. Porcentaje de estudiantes de universidades del CRUCH y universidades privadas por año según condición de matrícula, titulación, deserción y reingreso de los graduados de educación secundaria en 2006 que ingresaron a la educación superior en 2007.

Fuente: Elaboración propia sobre la base de Mineduc 2006 y SIES (2007 - 2015).

De modo análogo al sector técnico profesional, se calcularon perfiles estadísticos para el cuarto, séptimo y noveno año (Anexo, Tablas 1 G y $1 \mathrm{H}$ ). Para el sector universitario en su conjunto, y en todos los años, los estudiantes titulados, matriculados y reingresantes del mismo sector tienen mayor ranking que los desertores y que los reingresantes técnicos. Baja escolaridad de la madre se asocia a deserción y reingreso técnico profesional, mientras los matriculados y reingresantes universitarios se asocian a mayores niveles de ingreso, mayor proporción de mujeres y más altos puntajes PSU. Las ayudas 
estudiantiles, como se ha observado en la literatura, protegen de la deserción, favorecen la titulación y ahora, con estos resultados, se sabe que favorecen también el reingreso universitario. El hecho de que los estudiantes de mayor nivel socioeconómico tiendan a reingresar más a la universidad podría asociarse a menores barreras de entrada a distintas alternativas académicas y mejores posibilidades de encontrar un buen match.

Tabla 5

Perfil estadistico de estudiantes universitarios de los graduados de educación secundaria en 2006 que ingresaron a la educación superior en 2007 al séptimo año según condición de deserción, permanencia y reingreso técnico o universitario

\begin{tabular}{lccccc}
\hline & Desertor & Matriculado & $\begin{array}{c}\text { Reingresante Reingresante } \\
\text { técnico }\end{array}$ & Titulado \\
universitario & Tito \\
\hline Ranking notas colegio & $53 \%$ & $65 \%$ & $46 \%$ & $54 \%$ & $64 \%$ \\
\hline PSU promedio & 523 & 598 & 508 & 570 & 575 \\
\hline Modalidad TP & $21 \%$ & $9 \%$ & $18 \%$ & $9 \%$ & $13 \%$ \\
\hline P. Pagado & $15 \%$ & $26 \%$ & $13 \%$ & $28 \%$ & $27 \%$ \\
\hline P. Subvencionado & $46 \%$ & $46 \%$ & $50 \%$ & $47 \%$ & $44 \%$ \\
\hline Escolaridad madre & 12,1 & 13,0 & 12,0 & 13,2 & 12,8 \\
\hline Ingreso familiar en US\$ & 1.077 & 1.440 & 1.001 & 1.540 & 1.513 \\
\hline Mujer & $48 \%$ & $44 \%$ & $45 \%$ & $46 \%$ & $60 \%$ \\
\hline Ayuda estudiantil & $24 \%$ & $34 \%$ & $23 \%$ & $24 \%$ & $29 \%$ \\
\hline
\end{tabular}

Fuente: Elaboración propia sobre la base de Mineduc 2006 y SIES (2007 - 2015).

$\mathrm{Al}$ analizar los perfiles de cada una de los tipos de trayectoria académica en la educación superior universitaria, es claro que mejores condiciones académicas y socioeconómicas favorecen la titulación y permanencia, además de probabilizar el reingreso universitario, mientras que los estudiantes más vulnerables tienden concentrarse en la condición de desertor o bien de reingresante a una alternativa técnico profesional. El reingreso universitario, por tanto, se asocia a perfiles opuestos a los que determinarían la deserción universitaria. 


\subsection{Análisis de la titulación}

Los resultados descriptivos a continuación hacen absolutamente necesaria una revisión del concepto de deserción como fracaso académico. Si, por el contrario, se considera a la titulación como indicativa de éxito académico y se incorpora el reingreso entre las trayectorias posibles, los resultados de este estudio indican que más del 60\% de los estudiantes de la educación superior en Chile termina titulándose al noveno año, a pesar de los significativos niveles de deserción. Estos resultados desafían las aproximaciones teóricas y empíricas tradicionales, al tiempo que exigen superar la visión binaria de éxito académico y complejizar la teoría de las trayectorias en educación superior.

Metodológicamente, se procedió a analizar las tasas de titulación al cuarto, séptimo y noveno año de los estudiantes según su estatus académico en el tercer año: desertor, reingresante (según tipo de institución y área del conocimiento) y matriculado. Se analizó la titulación al noveno año para ampliar el horizonte de tiempo y equilibrar las diferencias generadas por la mayor duración de las carreras universitarias. Las tasas de titulación al cuarto y séptimo año pueden ser revisadas en el Anexo en las Tablas 1K y $1 \mathrm{~L}$.

En la Tabla 9 se muestran las tasas de titulación al noveno año. Se observa que el reingreso al sector técnico profesional —luego de la deserción universitaria - en promedio supera las tasas de titulación superiores de los estudiantes que reingresan al sector universitario. Un patrón algo menos claro se observa en el sector técnico profesional, donde el reingreso universitario de desertores a distinta área del conocimiento reduce las tasas de titulación significativamente, respecto de quedarse en el mismo tipo de institución y misma área de estudio. 
164 MÁS ALLÁ DE LA DESERCIÓN: TRAYECTORIAS ACADÉMICAS EN LA EDUCACIÓN SUPERIOR EN CHILE - Ch. Blanco, F. Meneses y R. Paredes

Tabla 6

Porcentaje de estudiantes titulados al noveno año, según su estatus en tercer año

\begin{tabular}{lcccccc}
\hline $\begin{array}{l}\text { Instituciones } \\
\text { originales }\end{array}$ & Desertor & $\begin{array}{c}\text { Reingreso U } \\
\text { otra área }\end{array}$ & $\begin{array}{c}\text { Reingreso U } \\
\text { misma área }\end{array}$ & $\begin{array}{c}\text { Reingreso } \\
\text { TC otra área }\end{array}$ & $\begin{array}{c}\text { Reingreso } \\
\text { TC misma } \\
\text { área }\end{array}$ & Matrícula \\
\hline CRUCH & $23 \%$ & $46 \%$ & $52 \%$ & $67 \%$ & $63 \%$ & $73 \%$ \\
Privadas & $24 \%$ & $51 \%$ & $54 \%$ & $52 \%$ & $56 \%$ & $71 \%$ \\
Universidades & $24 \%$ & $53 \%$ & $52 \%$ & $58 \%$ & $60 \%$ & $72 \%$ \\
CFT & $19 \%$ & $0 \%$ & $53 \%$ & $60 \%$ & $49 \%$ & $64 \%$ \\
IP & $16 \%$ & $50 \%$ & $49 \%$ & $32 \%$ & $51 \%$ & $70 \%$ \\
Técnicos & $17 \%$ & $46 \%$ & $57 \%$ & $51 \%$ & $50 \%$ & $68 \%$ \\
\hline
\end{tabular}

Nota: Cifras en negritas se refieren al promedio de titulación al noveno año, para totas las Universidades y para todos los CFTs e IPS.

Fuente: Elaboración propia sobre la base de Mineduc 2006 y SIES (2007 - 2015).

Aunque no logran alcanzar las tasas de titulación de quienes nunca desertaron, los reingresantes al tercer año logran tasas de titulación por sobre el 50\% una vez transcurridos nueve años. En las universidades del CRUCH, los estudiantes que reingresaron a otra área de estudio universitaria, obtuvieron tasas de titulación en torno al $46 \%$, en tanto quienes reingresaron a la misma área de estudios consiguieron tasas de titulación más altas, en torno al 52\%. Al parecer, mantenerse en el área de estudios favorece la titulación, en cualquier institución, lo cual sugiere una oportunidad de mejoramiento por medio de mayores posibilidades de articulación entre carreras de la misma área. A su vez, estudiantes del CRUCH que reingresaron al sector técnico profesional presentaron tasas de titulación de 10 puntos porcentuales por sobre sus pares en universidades privadas. Algo similar ocurrió en los casos de los IP, donde los estudiantes que reingresaron al sector universitario obtuvieron tasas de titulación tanto o más altas que los que reingresaron al sector técnico profesional.

En síntesis, en todo tipo de institución y para cualquier área de estudio, desertar no implica un fracaso académico. Cerca de la mitad de los desertores regresará a la educación superior en el corto plazo y la mitad habrá obtenido un título al noveno año. Existen diferencias significativas por tipo de institución y área del conocimiento, que interactúan con las tasas de titulación. Estas diferencias pueden ser estudiadas desde la perspectiva de la mismatch hypothesis con mayor 
éxito que desde perspectivas tradicionales que no pueden dar cuenta de trayectorias más complejas en educación superior.

Estudios ulteriores podrán profundizar incluso en diferencias por instituciones específicas, como las que también se revelan en este estudio (ver Anexo). Hay instituciones con muy altas tasas de titulación y reingreso, mientras que otras exhiben cifras mucho menores. Hay instituciones de carácter técnico profesional que superan en gran medida los niveles de titulación que otras de carácter universitario.

\section{Resultados predictivos}

Diferenciando entre los grupos técnico profesional y universitario, se realizaron dos análisis de regresión multinomial (fórmulas en Anexo). En una primera instancia - con propósito confirmatorio de lo reportado en la literatura - se predijo la condición de titulación, deserción o permanencia como matriculado al cuarto año. En la segunda etapa —con propósito exploratorio— se predijo el reingreso según tipo de institución y según área de estudio también al cuarto año. En ambos ejercicios, las variables independientes son las mismas con las que se construyeron los perfiles en la sección de estadísticas descriptivas.

\subsection{Estudiantes técnico profesionales}

\subsubsection{Primera etapa técnico profesionales}

Se confirma que la probabilidad de desertar de una institución técnico profesional al cuarto año disminuye a medida que aumenta el ranking de enseñanza media, el puntaje PSU, la ayuda financiera y si el estudiante es mujer. Por el contrario, la deserción aumenta si estudió en modalidad técnico profesional en la enseñanza media. La probabilidad de titularse disminuye si el estudiante proviene de la modalidad escolar técnico profesional en la enseñanza media y aumenta si el estudiante recibe ayuda financiera, es mujer o si tiene un alto ranking escolar o PSU. La probabilidad de permanecer matriculado aumenta con la PSU, el ingreso y, en menor medida, con las ayudas estudiantiles. El hecho de que ser mujer disminuya la 
probabilidad de permanencia se explica porque es más probable que al cuarto año una mujer ya se haya titulado. Los coeficientes y su nivel de significación estadística se presentan en la Tabla 1M en el Anexo.
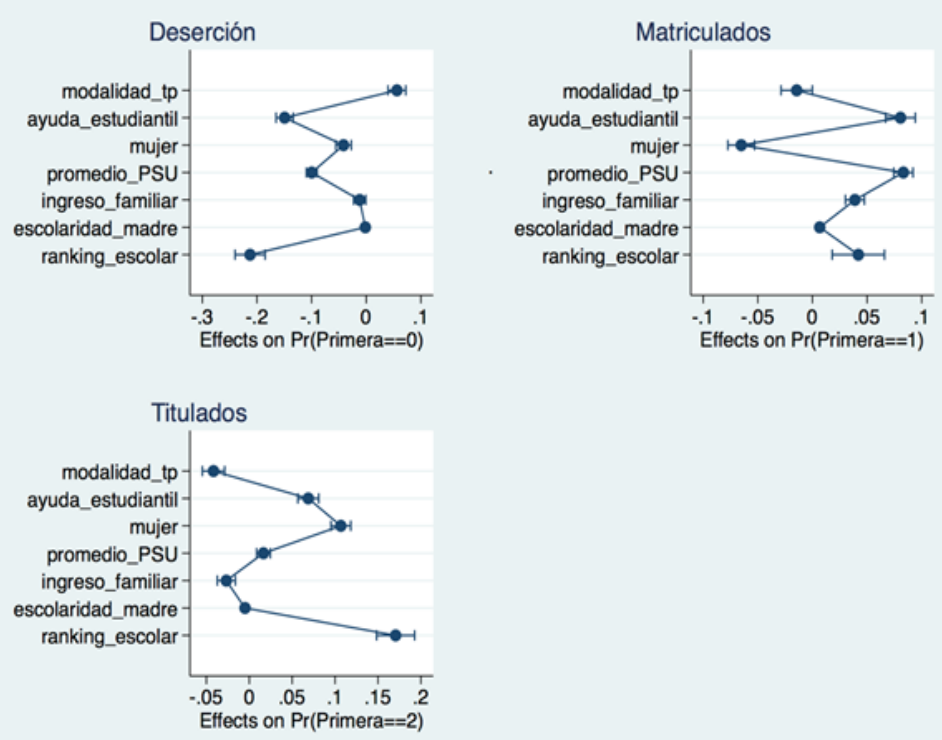

Figura 5. Resultados de regresiones de primera etapa: deserción, matrícula y titulación para estudiantes técnico profesionales.

Fuente: Elaboración propia sobre la base de Mineduc 2006 y SIES (2007 - 2015).

La Figura 5 muestra los coeficientes asociados a cada una de las variables. En la medida en que el punto se aleje más del cero en el eje horizontal, se trata de una variable con mayor impacto y este será significativo estadísticamente si los intervalos de confianza no incluyen el cero.

\subsubsection{Segunda etapa técnico profesionales}

Al predecir la deserción y los distintos tipos de reingreso en una regresión multinomial, se observan diferencias por tipo de institución y área de estudios. Un alto ranking escolar, alta PSU, ayuda financiera y ser mujer predice el reingreso a la educación universitaria y, específicamente, a misma área de conocimiento. Es posible que estudiantes con alto ranking hayan elegido la educación técnica en primera instancia, pero dadas sus capacidades de estudio, no hayan 
tenido un buen match con el programa seleccionado y decidan cambiarse a una carrera universitaria. La mismatch hypothesis podría tener buenos rendimientos explicativos para este fenómeno.

Estos inéditos resultados enriquecen los hallazgos de la primera etapa. De aquellos que desertaron en primera instancia, los que desertan definitivamente del sistema tienden a ser de la modalidad técnico profesional, no tener ayuda financiera, ser hombres, tener baja PSU, bajo nivel de ingresos y poseer un bajo ranking escolar. Por el contrario, quienes reingresan al sistema universitario poseen alto ranking, son en mayor proporción mujeres y cuentan con ayuda financiera.

Estos análisis de reingreso permiten una mejor caracterización de quienes son vulnerables a la doble deserción que, según sus perfiles ex ante, pueden ser identificados para hacerlos sujeto de programas remediales específicos o de orientación vocacional. Por ejemplo, los estudiantes de alto ranking escolar, las mujeres y quienes reciben ayuda financiera del Estado tienden a reingresar a la educación universitaria.

Lo anterior es importante para la política pública desde dos perspectivas. Primero, respecto del mismatch que se estaría dando en la educación técnico profesional entre estudiantes de alto ranking que tienden a migran al sector universitario, grupo al cual podría intervenirse con mejor información y orientación vocacional. Segundo, se visibiliza el impacto de la ayuda financiera en la elección de educación universitaria por sobre la educación técnico profesional. En efecto, la literatura nacional ya ha encontrado que la ayuda financiera generaría un sesgo a favor de la educación universitaria en desmedro de la educación técnico profesional (Blanco y Meneses, 2013) y estos resultados suman evidencia respecto del fenómeno. 


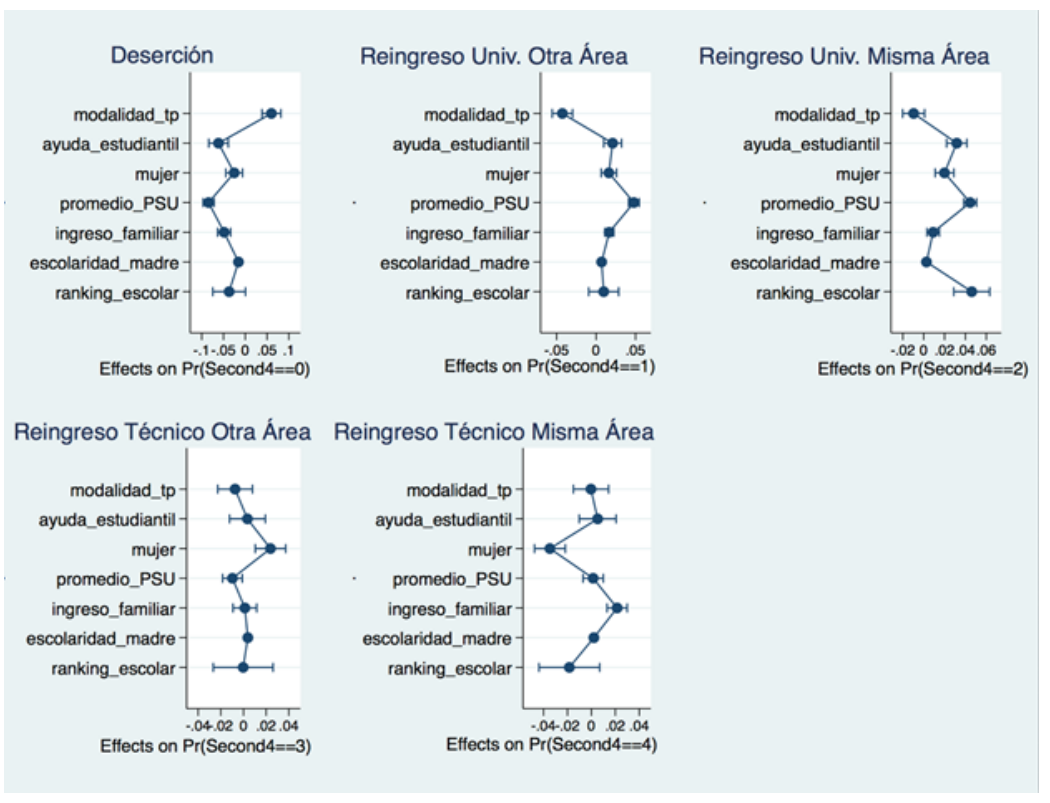

Figura 6. Resultados de regresiones de segunda etapa: reingreso para estudiantes técnico profesionales.

Fuente: Elaboración propia sobre la base de Mineduc 2006 y SIES (2007 - 2015).

Respecto de la concordancia del área de estudio inicial y de reingreso, aunque la mayoría de las variables no resultan significativas, el provenir de la modalidad científico humanista probabiliza el cambio de área en el reingreso universitario, al igual que tener alta PSU y alto nivel de ingreso. Para los reingresos técnicos, las variables predictoras no resultan significativas.

\subsection{Estudiantes universitarios}

Al igual que con los estudiantes técnico profesionales o vocacionales, se ejecutó el mismo análisis en dos etapas para los estudiantes universitarios. En la primera etapa se buscó verificar los efectos ya conocidos de las variables académicas, institucionales y sociodemográficas en la permanencia, deserción y titulación. En la segunda etapa, que constituye la mayor novedad de este estudio, se analizó el reingreso de los desertores universitarios según tipo de institución y área del conocimiento. 


\subsubsection{Primera etapa universitarios}

A partir de los resultados de las regresiones, se observa que la probabilidad de desertar de la universidad disminuye intensamente a medida que aumenta el ranking del colegio, aunque también al aumentar la PSU, la ayuda financiera o si se es mujer. Por el contrario, la probabilidad de desertar aumenta si el estudiante es de modalidad escolar técnico profesional.
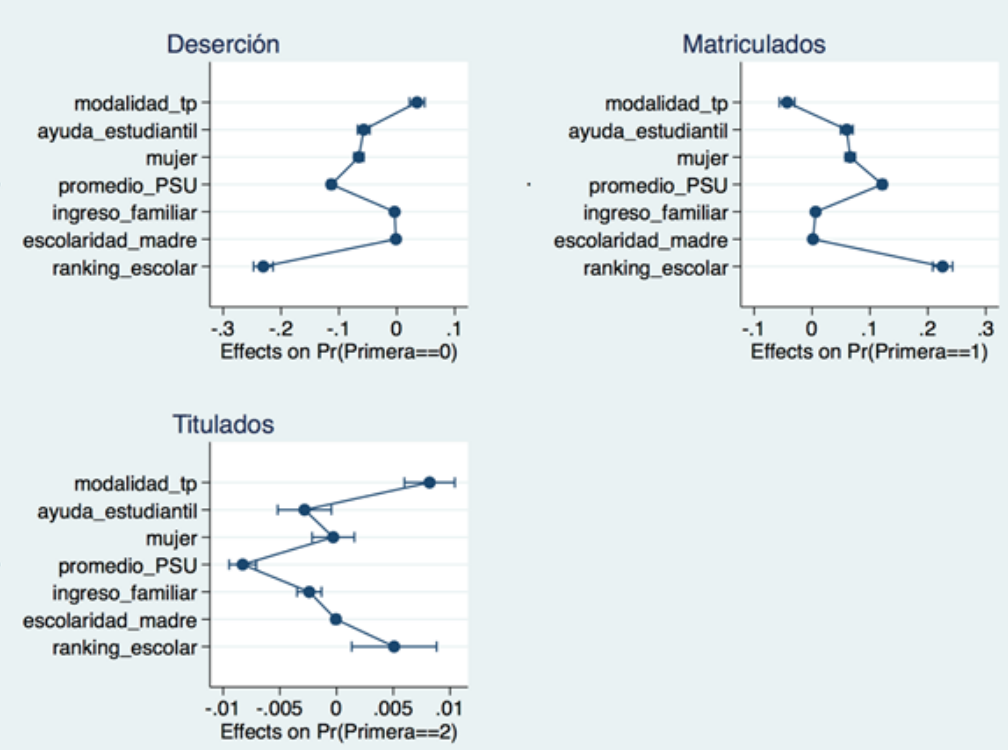

Figura 7. Resultados de regresiones de primera etapa: reingreso para universitarios.

Fuente: Elaboración propia sobre la base de Mineduc 2006 y SIES (2007 - 2015).

Un alto ranking escolar incrementa la probabilidad de mantenerse en la universidad, lo que también ocurre si el estudiante es mujer o si cuenta con ayuda financiera. Los resultados para titulación son menos relevantes y significativos, debido al bajo número de estudiantes titulados al cuarto año.

\subsubsection{Segunda etapa universitarios}

El análisis del reingreso revela importantes diferencias por tipo de educación (técnico profesional o universitaria) y área de estudios. Un alto ranking escolar y la presencia de ayuda financiera disminuyen la 
probabilidad de reingreso a la educación técnico profesional desde la universitaria, aumentando la probabilidad de reingreso al sector universitario.
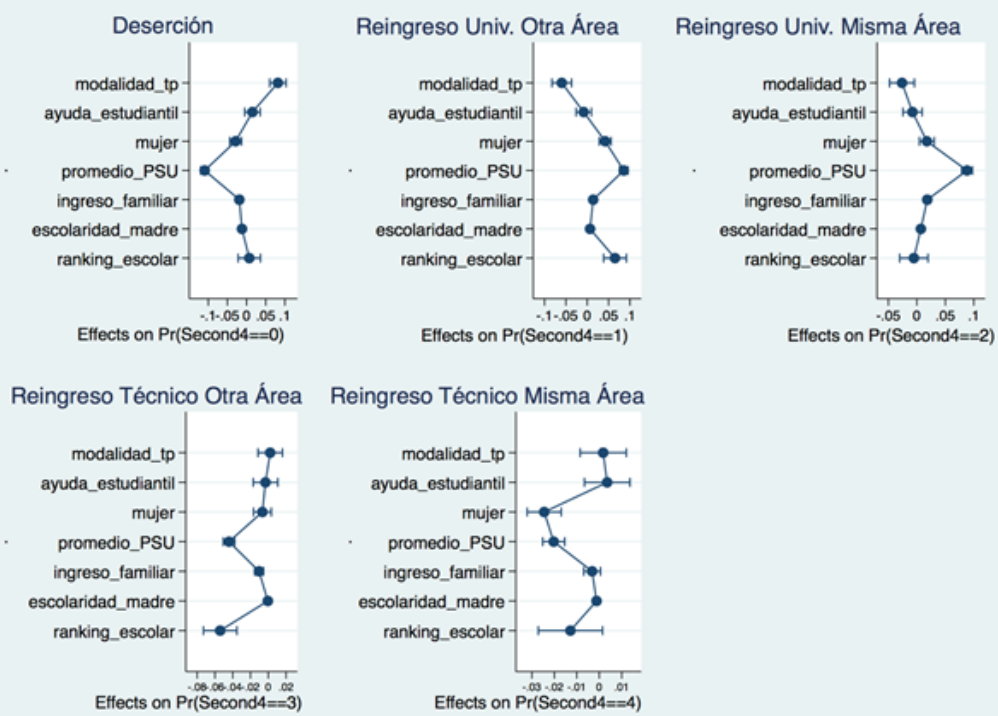

Reingreso Técnico Misma Área

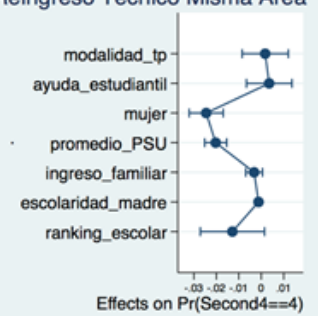

Figura 8. Resultados de regresiones de segunda etapa: reingreso para universitarios.

Fuente: Elaboración propia sobre la base de Mineduc 2006 y SIES (2007 - 2015).

Por otro lado, menor ranking escolar se asocia a reingreso al sector técnico profesional. Se aprecia además que provenir de la modalidad técnico profesional aumenta la probabilidad de la deserción del sistema, en tanto esta deserción disminuye a medida en que aumenta el nivel de ingresos y de puntaje PSU.

Los resultados del análisis predictivo de reingreso de estudiantes universitarios otorgan importante evidencia que puede ser interpretada desde las teorías del mismatch, en la medida en que los perfiles asociados al ingreso universitario inicial se corresponden con los del reingreso universitario. 


\section{Consideraciones finales}

La retención de estudiantes en sus carreras y la finalización de estudios en la educación superior se consideran habitualmente como objetivos primordiales en sistemas educativos efectivos. Lo contrario, la deserción, es habitualmente identificada con el fracaso académico. Probablemente por este motivo —y también por disponibilidad de datos- prevalece una visión binaria que reduce la evaluación de las trayectorias académicas a la permanencia y la deserción. Este trabajo innova al realizar un seguimiento de las trayectorias de los estudiantes en la educación superior, analizando su reingreso. La mitad de aquellos que desertan el primer año, reingresará a alguna alternativa de educación superior y su destino estará nuevamente cruzado por una serie de condiciones académicas, institucionales y sociodemográficas, aun cuando muchos de ellos llegarán a titularse en su segundo ingreso al sistema.

Los resultados indican que después de nueve años, uno de cada cuatro estudiantes se ha retirado del sistema para no volver, mientras que un $60 \%$ se ha titulado y un $15 \%$ continúa estudiando. Esto revela que si bien el sistema de educación superior es ineficiente en términos del tiempo de estudios - comparado con algunas métricas internacionales - es flexible y efectivo en el sentido de que es capaz de volver a captar estudiantes que desean reingresar y, finalmente, titula a un porcentaje significativo. La diversidad institucional, de tipo de educación y de áreas del conocimiento puede estar detrás de estas cifras. Sin embargo, a la vez puede ser la causa de los mismatch de los ingresos iniciales, en la medida en que la información está dispersa, fragmentada y puede que no permita un proceso de toma de decisiones más reflexivo que resulte en un mejor ajuste al matricularse.

Aunque ligeramente más en las universidades que en los CFT e IP, aproximadamente dos de cada tres de los reingresantes lo hacen en el mismo tipo de institución de la cual desertaron. En contraste, más del $50 \%$ de los estudiantes que reingresa a la educación superior, lo hace a un área de estudios distinta en la cual estuvo originalmente, lo que evidencia que la deserción tendría un componente relacionado 
con la orientación vocacional, lo que generaría insatisfacciones con la experiencia del primer año.

A partir del tercer año, los reingresos a la educación superior comienzan a ser en la distinta área de estudios en mayor medida, lo que tendría relación con el matching entre el estudiante y su carrera, institución o tipo de educación.

Las tasas de titulación de los estudiantes reingresantes están en torno al $50 \%$, pero nunca igualan a las tasas de titulación de los estudiantes que se mantienen en sus carreras originales, incluso después de nueve años. La menor diferencia se ve en estudiantes que estaban originalmente en el sector universitario y se mueven al sector técnico profesional, evidenciando un mejor match, o bien, un comportamiento acorde a carreras más cortas y que acelerarían el ingreso al mercado laboral. La dimensión informacional de este patrón también debe ser abordada en investigaciones futuras.

Si bien los análisis agregados delinean tendencias y patrones, a la vez exigen una mirada específica caso a caso. Al analizar por institución, se verifica una gran diversidad tanto en el sector técnico profesional como universitario. Existen instituciones técnicas que presentan tasas de titulación y retención incluso más altas que varias universidades privadas, lo que podría hablar de una mayor eficiencia, cruzada con diferencias en la duración de las carreras.

Las regresiones corroboran la importancia de las ayudas estudiantiles y otros factores como el puntaje PSU y, especialmente, el ranking escolar para explicar la deserción y la permanencia, pero también agrega evidencia respecto de su papel en favorecer el reingreso a la educación superior. En particular, aquellos estudiantes que acceden al sector técnico profesional con alto ranking escolar y alta PSU tienden a dirigirse al sector universitario en caso de desertar. En el sector universitario ocurre un efecto inverso, ya que los estudiantes de menor ranking y menor PSU tenderían a reingresar al sector técnico profesional. Esto se asocia con un reajuste de perfiles homólogo a lo que ocurre en la matrícula inicial en la educación superior. 
Las altas tasas de deserción generan ineficiencias en el sistema de educación superior. Sin embargo, la pérdida social es inferior a la sugerida por estudios que no siguen los estudiantes por nueve años y no analizan reingreso. El reingreso no es un fracaso educacional, sino que en muchos casos se trata de un reajuste, donde los estudiantes reingresarían a áreas o instituciones donde sería un mejor match.

Para evaluar el reingreso de una manera integral debe considerarse también los costos que significa para el Estado, en particular para los sistemas de ayudas estudiantiles y la política de gratuidad. Así mismo, hay costos para las instituciones y para los estudiantes y sus familias, además de los costos de oportunidad de los recursos involucrados, temas que también deben ser objeto de examinación en estudios venideros. A su vez, parece necesario analizar la introducción de programas de orientación vocacional y apoyo a la diversidad a nivel nacional, para minimizar los costos asociados al reingreso.

La profundización —cuantitativa y cualitativa - en esta línea será necesaria para ofrecer explicaciones más satisfactorias a los patrones de reingreso observados y las distintas trayectorias académicas. Futuras investigaciones deberán incorporar distinciones como el momento de la deserción, más allá de la que ocurre en primer año, así como un análisis de los tiempos de titulación y la profundización de los perfiles asociados a las distintas trayectorias académicas, incluso vinculándolos con la articulación con el mercado laboral.

Investigaciones posteriores en esta línea pueden profundizar a nivel de carrera y no solo en cuanto al área del conocimiento. Estos modelos explicativos futuros podrían incluir, por ejemplo, la capacidad de retención de la carrera en la institución o el puntaje PSU promedio de quienes acceden. Asimismo, podría considerarse la diferencia entre el puntaje PSU de ingreso a la carrera y el promedio de ingreso de la generación, o un indicador de ranking de ingreso a la carrera, quizás más allá del ranking de enseñanza media. También podrían analizarse si hay una relación cuadrática cuando hay valores muy altos en ranking escolar. 
Nuevas variables pueden construirse con los datos disponibles, para ahondar el análisis entre match entre estudiante y carrera elegida. Incluso, podría avanzarse en un análisis que incluya las preferencias de postulación de los estudiantes y la retención o graduación oportuna de las carreras. Además, variables complementarias del establecimiento de enseñanza media, por ejemplo, resultados en pruebas estandarizadas o si los estudiantes contaron con orientación vocacional podrían otorgar nuevas luces al fenómeno en cuestión. Incluso, sería posible incorporar la dimensión geográfica, en tanto mayores distancias a las instituciones de educación superior podrían asociarse a mayor deserción.

La necesidad de sofisticar la mirada teórica y la superación de perspectivas binarias y reduccionistas constituye el mayor aporte teórico y metodológico de este estudio. Simultáneamente, la política pública puede y debe servirse de estos resultados, en la medida en que no hacerlo significaría ignorar la especificidad de una enorme cantidad de estudiantes que, por diversos motivos, desertaron en primer año y vuelven al sistema en búsqueda de una nueva oportunidad. Una perspectiva de política pública basada en la evidencia deberá considerar medidas preventivas y remediales para esta nueva realidad que se abre a los investigadores en educación superior.

\section{Referencias}

Ajzen, I., \& Fishbein, M. (1977). Attitude-behavior relations: A theoretical analysis and review of empirical research. Psychological Bulletin, 84(5), 888 .

Arcidiacono, P., \& Lovenheim, M. (2016). Affirmative action and the qualityfit trade-off. Journal of Economic Literature, 54(1), 3-51.

Bailey, T., Jeong, D. W., \& Cho, S.-W. (2010). Referral, enrollment, and completion in developmental education sequences in community colleges. Economics of Education Review, 29(2), 255-270.

Blanco, C., \& Meneses, F. (2013). Impacto de la ayuda financiera en la matrícula técnica y universitaria. Sociedad Hoy, (24).

Bordón, P., Canals, C., \& Rojas, S. (2015). Retención en los programas e instituciones de educación superior: nueva evidencia para Chile. Estudios de Política Educativa, 2, 176-214. 
Calcagno, J. C., Bailey, T., Jenkins, D., Kienzl, G., \& Leinbach, T. (2008). Community college student success: What institutional characteristics make a difference? Economics of Education Review, 27(6), 632-645.

Catrileo, C., Lobos, P., \& Sereño, F. (1991). Propedéutico UCSH, un programa de acceso inclusivo a la educación superior. Contexto, Experiencias e Investigaciones Sobre Los Programas Propedéuticos En Chile, 69.

Contreras, D., Gallegos, S., \& Meneses, F. (2009). Determinantes de desempeño universitario:? Importa la habilidad relativa? Calidad En La Educación, (30).

Devés, R., \& Mora-Curriao, M. (2014). Transformative Policies for Equity and Inclusion at the University of Chile. In Leadership for Social Justice in Higher Education (pp. 85-101). Springer.

Díaz Peralta, C. (2008). Modelo conceptual para la deserción estudiantil universitaria chilena. Estudios Pedagógicos (Valdivia), 34(2), 65-86.

Farías, M., \& Sevilla, M. P. (2015). Effectiveness of Vocational High Schools in Students' Access to and Persistence in Postsecondary Vocational Education. Research in Higher Education, 56(7), 693-718.

Gallardo, G., Lorca, A., Morrás, D., \& Vergara, M. (2014). Experiencia de transición de la secundaria a la universidad de estudiantes admitidos en una universidad tradicional chilena (CRUCH) vía admisión especial de carácter inclusivo. Pensamiento Educativo, 51(2), 135-151.

Goldrick-Rab, S. (2010). Challenges and opportunities for improving community college student success. Review of Educational Research, 80(3), 437-469.

González, L. E., \& Uribe, D. (2002). Estimaciones sobre la "repitencia" y deserción en la educación superior chilena. Consideraciones sobre sus implicaciones. Revista Calidad En La Educación Consejo Superior de Educación Diciembre Del, 2002, 77.

Larrañaga, O., Cabezas, G., \& Dussaillant, F. (2014). Trayectorias educacionales e inserción laboral en la enseñanza media técnico profesional. Estudios Públicos, 134, 7-58.

Melguizo, T., Sanchez, F., \& Velasco, T. (2016). Credit for low-income students and access to and academic performance in higher education in Colombia: A regression discontinuity approach. World Development, $80,61-77$.

Melguizo, T., Torres, F. S., \& Jaime, H. (2011). The association between financial aid availability and the college dropout rates in Colombia. Higher Education, 62(2), 231-247. 
Ministerio de Desarrollo Social. (2015). CASEN 2015.

Rolando, R., Salamanca, J., Lara, A., \& Blanco, C. (2012a). Deserción \& reingreso a educación superior en Chile. Santiago: Sies, Mifuturo. $\mathrm{Cl}$.

Rolando, R., Salamanca, J., Lara, A., \& Blanco, C. (2012b). Deserción y reingreso a Educación Superior en Chile. Análisis de la Cohorte 2008. Santiago de Chile: Servicio de Información de Educación Superior-[SIES] [Links].

Sander, R. H. (2004). A systemic analysis of affirmative action in American law schools. Stan. L. Rev., 57, 367.

Santelices, M. V., Catalán, X., Kruger, D., \& Horn, C. (2016). Determinants of persistence and the role of financial aid: lessons from Chile. Higher Education, 71(3), 323-342.

Spady, W. G. (1970). Dropouts from higher education: An interdisciplinary review and synthesis. Interchange, 1(1), 64-85.

Stratton, L. S., O’Toole, D. M., \& Wetzel, J. N. (2008). A multinomial logit model of college stopout and dropout behavior. Economics of Education Review, 27(3), 319-331.

SIES, S. de I. de E. (2015). Panorama de la educación superior en Chile 2014. Mineduc Santiago de Chile.

Tinto, V. (1975). Dropout from higher education: A theoretical synthesis of recent research. Review of Educational Research, 45(1), 89-125.

Tinto, V. (1987). Leaving college: Rethinking the causes and cures of student attrition. ERIC.

Tracey, B., \& Florian, K. (2016). Educational Research and Innovation Governing Education in a Complex World. OECD Publishing.

Yorke, M., \& Longden, B. (2004). Retention and student success in higher education. McGraw-Hill Education (UK). 


\section{Anexo}

Las regresiones Mlogit para categorías se describen a continuación. Para ello, se opera según la siguiente regresión, que se repite para cada uno de los grupos:

Resultado $_{i}(1.1,1.2,1.3)=\beta_{0}+\beta_{1 j}$ Académicas $+\beta_{2 j}$ Institucionales $+\beta_{3 j}$ Sociodemográficas

Las $\mathrm{j}$ variables explicativas se agruparon en variables académicas, socioeconómicas e institucionales y describiendo a las i estudiantes. Se usaron multinomiales logit para explicar el rendimiento de los estudiantes. Para analizar las diferencias de perfiles de transición, se realizaron regresiones por separado para estudiantes que comienzan en el sector técnico profesional y estudiantes que comienzan en el sector universitario.

Resultado $(2.1,2.2,2.3,2.4,2.5)=\beta_{0}+\beta_{1 i}$ Académicas $+\beta_{2 i}$ Institucionales $+\beta_{3 i}$ Sociodemográficas

Dimensionalidad de los determinantes de las trayectorias de educación superior:

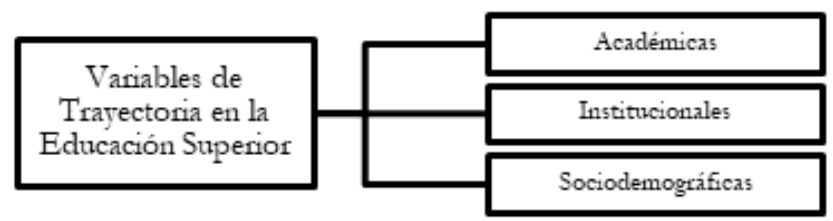

Figura 1A. Dimensionalidad de las trayectorias académicas en la educación superior para el presente estudio.

Fuente: Elaboración propia. 
178 MÁS ALLÁ DE LA DESERCIÓN: TRAYECTORIAS ACADÉMICAS EN LA EDUCACIÓN SUPERIOR EN CHILE - Ch. Blanco, F. Meneses y R. Paredes

Tabla $1 \mathrm{~A}$

Variables del estudio: dimensión académica

\begin{tabular}{lll}
\hline Variable & Fuente & Descripción \\
\hline Ranking & Mineduc & Ranking del estudiante en su colegio de origen. \\
Puntaje PSU & DEMRE & $\begin{array}{l}\text { Puntaje PSU promedio del estudiante de lenguaje y } \\
\text { matemática }\end{array}$ \\
Trayectoria & SIES & Matriculado, desertor, titulado o reingresante.
\end{tabular}

Fuente: Elaboración propia.

Tabla 1B

Variables del estudio: dimensión institucional

\begin{tabular}{|c|c|c|}
\hline Variable & Fuente & Descripción \\
\hline $\begin{array}{l}\text { Modalidad } \\
\text { educación media }\end{array}$ & Mineduc & $\begin{array}{l}\text { Modalidad educación media científico humanista o } \\
\text { técnico profesional. }\end{array}$ \\
\hline Dependencia & Mineduc & $\begin{array}{l}\text { Dependencia establecimiento: municipal, particular } \\
\text { subvencionado o particular pagado. }\end{array}$ \\
\hline Tipo de institución & SIES & Universidad (CRUCH o privada), CFT o IP. \\
\hline Tipo de educación & SIES & Educación universitaria o técnico profesional \\
\hline Área de estudio & SIES & Según clasificación OCDE (SIES 2016). \\
\hline
\end{tabular}

Fuente: Elaboración propia.

Tabla 1C

Variables del estudio: dimensión institucional

\begin{tabular}{|c|c|c|}
\hline Variable & Fuente & Descripción \\
\hline Escolaridad madre & DEMRE & Años de escolaridad de la madre. \\
\hline Ingreso mensual & DEMRE & Ingreso mensual familiar, medido en US\$. \\
\hline Mujeres & Mineduc & Si el estudiante es hombre o mujer. \\
\hline Ayuda estudiantil & SIES & $\begin{array}{l}\text { Si el estudiante tiene ayuda financiera estatal (crédito } \\
\text { o beca). }\end{array}$ \\
\hline
\end{tabular}

Fuente: Elaboración propia. 
Tabla 1D

Condición de matrícula, titulación y deserción de los egresados de educación secundaria en 2006 que ingresaron a la educación superior en 2007

\begin{tabular}{lccc}
\hline & Número estudiantes & Rinden PSU & Porcentaje \\
\hline CFT & 11.331 & 8.310 & $73 \%$ \\
IP & 18.892 & 16.027 & $85 \%$ \\
U. CRUCH & 31.731 & 31.495 & $99 \%$ \\
U. Privada & 26.447 & 25.667 & $97 \%$ \\
Ingresa a la ES & 88.401 & 81.499 & $92 \%$ \\
No ingresa a la ES & 150.565 & 65.888 & $44 \%$ \\
Total & 238.966 & 147.387 & $62 \%$ \\
\hline
\end{tabular}

Fuente: Elaboración propia sobre la base de Mineduc 2006 y SIES (2007 - 2015).

Tabla 1E 1

Cantidad de estudiantes por año según condición de matrícula, titulación y deserción de los graduados de educación secundaria en 2006 que ingresaron a la educación superior en 2007

\begin{tabular}{|c|c|c|c|c|c|c|c|c|}
\hline & \multicolumn{4}{|c|}{ Universitarios } & \multicolumn{4}{|c|}{ Técnicos } \\
\hline 安 & $\begin{array}{l}\frac{\pi}{3} \\
\stackrel{\Xi}{\Xi} \\
\sum\end{array}$ & 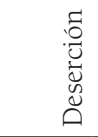 & 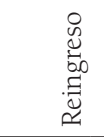 & 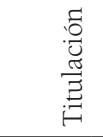 & 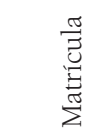 & 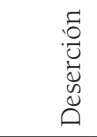 & 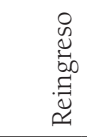 & 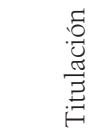 \\
\hline 1 & 58.178 & - & - & - & 30.223 & - & - & - \\
\hline 2 & 47.110 & 5.541 & 5.527 & - & 19.687 & 7.480 & 3.056 & - \\
\hline 3 & 40.983 & 6.714 & 10.321 & 160 & 11.464 & 9.640 & 5.419 & 3.700 \\
\hline 4 & 37.479 & 7.161 & 12.532 & 1.006 & 6.965 & 10.635 & 6.234 & 6.389 \\
\hline 5 & 31.806 & 7.575 & 13.370 & 5.427 & 3.293 & 11.271 & 5.885 & 9.774 \\
\hline 6 & 21.677 & 9.789 & 12.666 & 14.046 & 1.648 & 11.647 & 4.974 & 11.954 \\
\hline 7 & 12.258 & 10.674 & 11.326 & 23.920 & 773 & 11.674 & 4.129 & 13.647 \\
\hline 8 & 6.384 & 11.105 & 8.989 & 31.700 & 385 & 11.561 & 3.397 & 14.880 \\
\hline 9 & 3.320 & 11.178 & 6.714 & 36.966 & 206 & 11.431 & 2.676 & 15.910 \\
\hline
\end{tabular}

Fuente: Elaboración propia sobre la base de Mineduc 2006 y SIES (2007 - 2015). 
180 MÁS ALLÁ DE LA DESERCIÓN: TRAYECTORIAS ACADÉMICAS EN LA EDUCACIÓN SUPERIOR EN CHILE - Ch. Blanco, F. Meneses y R. Paredes

Tabla 1E 2

Porcentaje de estudiantes por año según condición de matrícula, titulación y deserción de los graduados de educación secundaria en 2006 que ingresaron a la educación superior en 2007

\begin{tabular}{|c|c|c|c|c|c|c|c|c|}
\hline \multirow[b]{2}{*}{ 是 } & \multicolumn{4}{|c|}{ Universitarios } & \multicolumn{4}{|c|}{ Técnicos } \\
\hline & $\begin{array}{l}\frac{\pi}{J} \\
\tilde{U} \\
\underbrace{\pi} \\
\sum\end{array}$ & 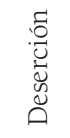 & 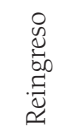 & 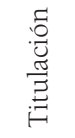 & $\begin{array}{l}\frac{\pi}{3} \\
\stackrel{\mathscr{\Xi}}{\Xi} \\
\stackrel{\pi}{\Sigma}\end{array}$ & 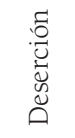 & 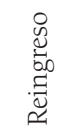 & 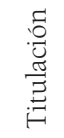 \\
\hline 1 & $100 \%$ & $0 \%$ & $0 \%$ & $0 \%$ & $100 \%$ & $0 \%$ & $0 \%$ & $0 \%$ \\
\hline 2 & $81 \%$ & $10 \%$ & $10 \%$ & $0 \%$ & $65 \%$ & $25 \%$ & $10 \%$ & $0 \%$ \\
\hline 3 & $70 \%$ & $12 \%$ & $18 \%$ & $0 \%$ & $38 \%$ & $32 \%$ & $18 \%$ & $12 \%$ \\
\hline 4 & $64 \%$ & $12 \%$ & $22 \%$ & $2 \%$ & $23 \%$ & $35 \%$ & $21 \%$ & $21 \%$ \\
\hline 5 & $55 \%$ & $13 \%$ & $23 \%$ & $9 \%$ & $11 \%$ & $37 \%$ & $19 \%$ & $32 \%$ \\
\hline 6 & $37 \%$ & $17 \%$ & $22 \%$ & $24 \%$ & $5 \%$ & $39 \%$ & $16 \%$ & $40 \%$ \\
\hline 7 & $21 \%$ & $18 \%$ & $19 \%$ & $41 \%$ & $3 \%$ & $39 \%$ & $14 \%$ & $45 \%$ \\
\hline 8 & $11 \%$ & $19 \%$ & $15 \%$ & $54 \%$ & $1 \%$ & $38 \%$ & $11 \%$ & $49 \%$ \\
\hline 9 & $6 \%$ & $19 \%$ & $12 \%$ & $64 \%$ & $1 \%$ & $38 \%$ & $9 \%$ & $53 \%$ \\
\hline
\end{tabular}

Fuente: Elaboración propia sobre la base de Mineduc 2006 y SIES (2007 - 2015).

Tabla $1 F$

Perfil estadístico de estudiantes técnico profesionales de los graduados de educación secundaria en 2006 que ingresaron a la educación superior en 2007 al séptimo año según condición de deserción, permanencia y reingreso técnico o universitario

\begin{tabular}{lccccc}
\hline & Desertor & Matriculado & $\begin{array}{c}\text { Reingresante } \\
\text { técnico }\end{array}$ & $\begin{array}{c}\text { Reingresante } \\
\text { universitario }\end{array}$ & Titulado \\
\hline Ranking notas colegio & $42 \%$ & $42 \%$ & $41 \%$ & $44 \%$ & $53 \%$ \\
PSU Promedio & 419 & 451 & 424 & 470 & 451 \\
Modalidad TP & $35 \%$ & $30 \%$ & $34 \%$ & $26 \%$ & $29 \%$ \\
P. Pagado & $3 \%$ & $7 \%$ & $4 \%$ & $9 \%$ & $4 \%$ \\
P. Subvencionado & $33 \%$ & $48 \%$ & $40 \%$ & $45 \%$ & $42 \%$ \\
Escolaridad madre & 10,4 & 11,4 & 10,9 & 11,7 & 10,6 \\
Ingreso mensual US\$ & 581 & 750 & 612 & 857 & 632 \\
Mujer & $44 \%$ & $34 \%$ & $46 \%$ & $51 \%$ & $58 \%$ \\
Ayuda estudiantil & $25 \%$ & $31 \%$ & $24 \%$ & $28 \%$ & $43 \%$ \\
\hline
\end{tabular}

Fuente: Elaboración propia sobre la base de Mineduc 2006 y SIES (2007 - 2015). 
Tabla $1 G$

Perfil estadístico de estudiantes técnico profesionales de los graduados de educación secundaria en 2006 que ingresaron a la educación superior en 2007 al noveno año según condición de deserción, permanencia y reingreso técnico o universitario.

\begin{tabular}{lccccc}
\hline & Desertor & Matriculado & $\begin{array}{c}\text { Reingresante } \\
\text { técnico }\end{array}$ & $\begin{array}{c}\text { Reingresante } \\
\text { universitario }\end{array}$ & Titulado \\
\hline Ranking notas colegio & $42 \%$ & $43 \%$ & $40 \%$ & $45 \%$ & $52 \%$ \\
PSU Promedio & 418 & 441 & 428 & 460 & 451 \\
Modalidad TP & $35 \%$ & $31 \%$ & $33 \%$ & $29 \%$ & $30 \%$ \\
P. Pagado & $3 \%$ & $4 \%$ & $3 \%$ & $8 \%$ & $4 \%$ \\
P. Subvencionado & $33 \%$ & $47 \%$ & $38 \%$ & $46 \%$ & $43 \%$ \\
Escolaridad madre & 10,4 & 11,1 & 10,9 & 11,5 & 10,7 \\
Ingreso mensual US\$ & 588 & 613 & 589 & 795 & 647 \\
Mujer & $43 \%$ & $29 \%$ & $44 \%$ & $51 \%$ & $57 \%$ \\
Ayuda estudiantil & $24 \%$ & $32 \%$ & $23 \%$ & $27 \%$ & $41 \%$ \\
\hline
\end{tabular}

Fuente: Elaboración propia sobre la base de Mineduc 2006 y SIES (2007 - 2015).

Tabla $1 \mathrm{H}$

Perfil estadístico de universitarios de los graduados de educación secundaria en 2006 que ingresaron a la educación superior en 2007 al cuarto año según condición de deserción, permanencia y reingreso técnico o universitario

\begin{tabular}{lccccc}
\hline & Desertor & Matriculado & $\begin{array}{c}\text { Reingresante } \\
\text { técnico }\end{array}$ & $\begin{array}{c}\text { Reingresante } \\
\text { universitario }\end{array}$ & Titulado \\
\hline Ranking notas colegio & $50 \%$ & $65 \%$ & $46 \%$ & $53 \%$ & $60 \%$ \\
PSU Promedio & 503 & 585 & 500 & 565 & 509 \\
Modalidad TP & $24 \%$ & $11 \%$ & $19 \%$ & $10 \%$ & $31 \%$ \\
P. Pagado & $13 \%$ & $26 \%$ & $13 \%$ & $29 \%$ & $9 \%$ \\
P. Subvencionado & $44 \%$ & $45 \%$ & $51 \%$ & $45 \%$ & $48 \%$ \\
Escolaridad madre & 11,7 & 12,9 & 12,0 & 13,2 & 11,7 \\
Ingreso mensual US\$ & 960 & 1.478 & 1.003 & 1.599 & 844 \\
Mujer & $48 \%$ & $53 \%$ & $45 \%$ & $48 \%$ & $55 \%$ \\
Ayuda estudiantil & $23 \%$ & $40 \%$ & $24 \%$ & $33 \%$ & $48 \%$ \\
\hline
\end{tabular}

Fuente: Elaboración propia sobre la base de Mineduc 2006 y SIES (2007 - 2015). 
182 MÁS ALLÁ DE LA DESERCIÓN: TRAYECTORIAS ACADÉMICAS EN LA EDUCACIÓN SUPERIOR EN CHILE - Ch. Blanco, F. Meneses y R. Paredes

Tabla 11

Perfil estadístico de estudiantes universitarios de los graduados de educación secundaria en 2006 que ingresaron a la educación superior en 2007 al séptimo año según condición de deserción, permanencia y reingreso técnico o universitario

\begin{tabular}{lccccc}
\hline & Desertor & Matriculado & $\begin{array}{c}\text { Reingresante } \\
\text { técnico }\end{array}$ & $\begin{array}{c}\text { Reingresante } \\
\text { universitario }\end{array}$ & Titulado \\
\hline Ranking notas colegio & $53 \%$ & $65 \%$ & $46 \%$ & $54 \%$ & $64 \%$ \\
PSU Promedio & 523 & 598 & 508 & 570 & 575 \\
Modalidad TP & $21 \%$ & $9 \%$ & $18 \%$ & $9 \%$ & $13 \%$ \\
P. Pagado & $15 \%$ & $26 \%$ & $13 \%$ & $28 \%$ & $27 \%$ \\
P. Subvencionado & $46 \%$ & $46 \%$ & $50 \%$ & $47 \%$ & $44 \%$ \\
Escolaridad madre & 12,1 & 13,0 & 12,0 & 13,2 & 12,8 \\
Ingreso mensual US\$ & 1.077 & 1.440 & 1.001 & 1.540 & 1.513 \\
Mujer & $48 \%$ & $44 \%$ & $45 \%$ & $46 \%$ & $60 \%$ \\
Ayuda estudiantil & $24 \%$ & $34 \%$ & $23 \%$ & $24 \%$ & $29 \%$ \\
\hline
\end{tabular}

Fuente: Elaboración propia sobre la base de Mineduc 2006 y SIES (2007 - 2015).

Tabla 1J

Perfil estadístico de estudiantes universitarios de los graduados de educación secundaria en 2006 que ingresaron a la educación superior en 2007 al noveno año según condición de deserción, permanencia y reingreso técnico o universitario

\begin{tabular}{lccccc}
\hline & Desertor & Matriculado & $\begin{array}{c}\text { Reingresante } \\
\text { técnico }\end{array}$ & $\begin{array}{c}\text { Reingresante } \\
\text { universitario }\end{array}$ & Titulado \\
\hline Ranking notas colegio & $52 \%$ & $63 \%$ & $46 \%$ & $54 \%$ & $63 \%$ \\
PSU Promedio & 526 & 587 & 508 & 566 & 579 \\
Modalidad TP & $20 \%$ & $10 \%$ & $20 \%$ & $10 \%$ & $12 \%$ \\
P. Pagado & $16 \%$ & $21 \%$ & $11 \%$ & $23 \%$ & $28 \%$ \\
P. Subvencionado & $47 \%$ & $48 \%$ & $49 \%$ & $49 \%$ & $44 \%$ \\
Escolaridad madre & 12,1 & 12,8 & 11,9 & 13,0 & 13,0 \\
Ingreso mensual US\$ & 1.099 & 1.243 & 937 & 1.374 & 1.526 \\
Mujer & $45 \%$ & $42 \%$ & $42 \%$ & $44 \%$ & $56 \%$ \\
Ayuda estudiantil & $24 \%$ & $34 \%$ & $22 \%$ & $27 \%$ & $29 \%$ \\
\hline
\end{tabular}

Fuente: Elaboración propia sobre la base de Mineduc 2006 y SIES (2007 - 2015). 
Tabla $1 \mathrm{~K}$

Porcentaje de estudiantes titulados al cuarto año, según su estatus en tercer año

\begin{tabular}{lcccccc}
\hline $\begin{array}{l}\text { Instituciones } \\
\text { originales }\end{array}$ & Desertor & $\begin{array}{c}\text { Reingreso } \\
\text { U otra área }\end{array}$ & $\begin{array}{c}\text { Reingreso } \\
\text { U misma } \\
\text { área }\end{array}$ & $\begin{array}{c}\text { Reingreso } \\
\text { TC otra área }\end{array}$ & $\begin{array}{c}\text { Reingreso } \\
\text { TC misma } \\
\text { área }\end{array}$ & Matrícula \\
\hline CRUCH & $1 \%$ & $0 \%$ & $1 \%$ & $10 \%$ & $8 \%$ & $1 \%$ \\
Privadas & $1 \%$ & $1 \%$ & $0 \%$ & $6 \%$ & $7 \%$ & $1 \%$ \\
Universidades & $1 \%$ & $0 \%$ & $0 \%$ & $8 \%$ & $7 \%$ & $1 \%$ \\
CFT & $5 \%$ & $0 \%$ & $7 \%$ & $7 \%$ & $9 \%$ & $35 \%$ \\
IP & $2 \%$ & $1 \%$ & $3 \%$ & $13 \%$ & $8 \%$ & $11 \%$ \\
Técnicos & $3 \%$ & $1 \%$ & $4 \%$ & $11 \%$ & $8 \%$ & $17 \%$ \\
\hline
\end{tabular}

Notas: cifras en negritas se refieren al promedio de titulación al noveno año, para totas las Universidades y para todos los CFTs e IPS.

Fuente: Elaboración propia sobre la base de Mineduc 2006 y SIES (2007 - 2015).

Tabla 1L

Porcentaje de estudiantes titulados al séptimo año, según su estatus en tercer año

\begin{tabular}{lrccccc}
\hline $\begin{array}{l}\text { Instituciones } \\
\text { originales }\end{array}$ & Desertor & $\begin{array}{c}\text { Reingreso } \\
\text { U otra área }\end{array}$ & $\begin{array}{c}\text { Reingreso } \\
\text { U misma } \\
\text { área }\end{array}$ & $\begin{array}{c}\text { Reingreso } \\
\text { TC otra área }\end{array}$ & $\begin{array}{c}\text { Reingreso } \\
\text { TC misma } \\
\text { área }\end{array}$ & Matrícula \\
\hline CRUCH & $9 \%$ & $19 \%$ & $20 \%$ & $40 \%$ & $50 \%$ & $48 \%$ \\
Privadas & $11 \%$ & $28 \%$ & $27 \%$ & $39 \%$ & $42 \%$ & $51 \%$ \\
Universidades & $10 \%$ & $23 \%$ & $24 \%$ & $44 \%$ & $48 \%$ & $49 \%$ \\
CFT & $13 \%$ & $0 \%$ & $38 \%$ & $60 \%$ & $40 \%$ & $60 \%$ \\
IP & $8 \%$ & $40 \%$ & $28 \%$ & $21 \%$ & $42 \%$ & $61 \%$ \\
Técnicos & $10 \%$ & $26 \%$ & $37 \%$ & $42 \%$ & $40 \%$ & $61 \%$ \\
\hline
\end{tabular}

Notas: cifras en negritas se refieren al promedio de titulación al noveno año, para totas las Universidades y para todos los CFTs e IPS.

Fuente: Elaboración propia sobre la base de Mineduc 2006 y SIES (2007 - 2015).

\section{Resultados descriptivos: diferencias institucionales}

Existen amplias diferencias entre tipo de instituciones de educación superior y las instituciones de un mismo tipo. Mientras algunas muestran tasas de titulación sobre el $60 \%$, otras presentan tasas de titulación en torno al 20\% (Figura 1B). Al analizar los estudiantes matriculados inicialmente en las instituciones técnicas con mejores resultados, estos obtienen éxitos superiores a los de sus pares: mayores tasas de titulación, matrícula y reingreso técnico y universitario, superando incluso a varias universidades, 
fundamentalmente privadas, pero también que algunas del CRUCH. Por lo tanto, aunque no es el objetivo de este trabajo, futuras aproximaciones podrán identificar diferencias en las trayectorias de los estudiantes a nivel de institución y no solo por tipo de institución.

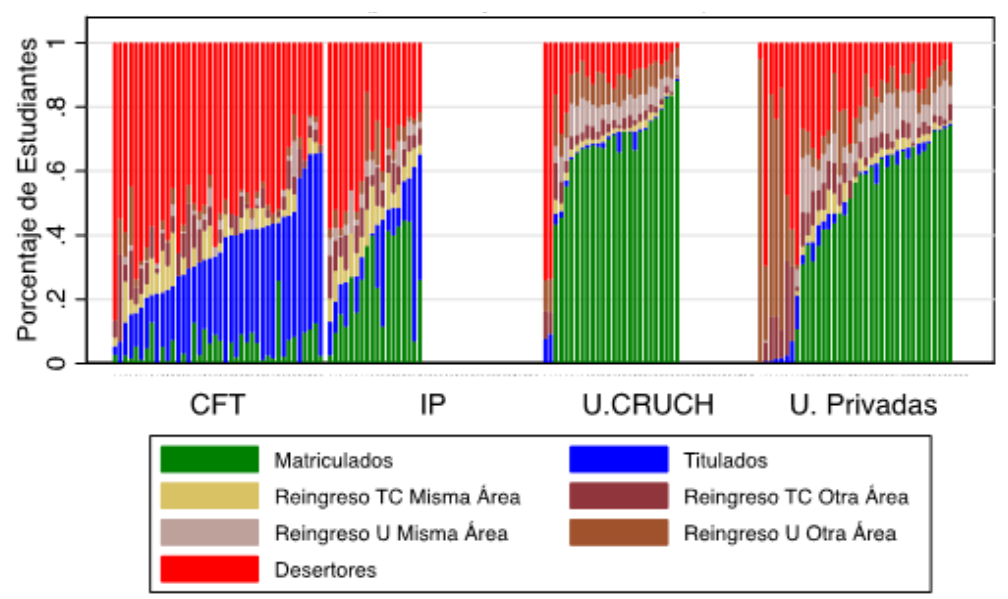

Figura 1B. Porcentaje de estudiantes por tipo de institución y por año según condición de matrícula, titulación, deserción y reingreso de los graduados de educación secundaria en 2006 que ingresaron a la educación superior en 2007.

Fuente: Elaboración propia sobre la base de Mineduc 2006 y SIES (2007 - 2015).

El hecho de que las carreras sean más largas en el mundo universitario, sumando a la mayor deserción en las instituciones técnicas, hace que predominen en los primeros años mayores niveles de matrícula en las universidades, especialmente en las CRUCH. 


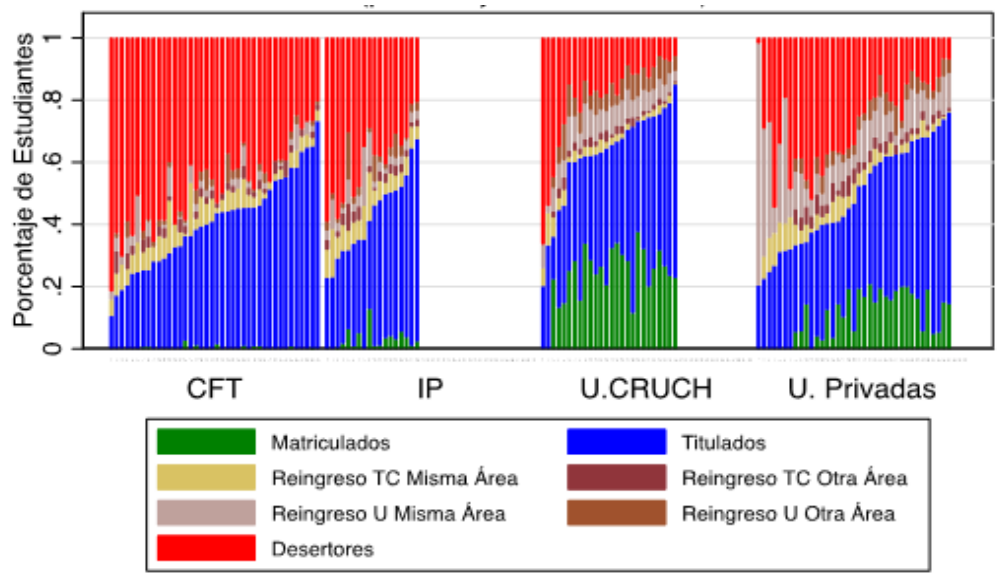

Figura 1C. Porcentaje de estudiantes por tipo de institución y por año según condición de matrícula, titulación, deserción y reingreso de los graduados de educación secundaria en 2006 que ingresaron a la educación superior en 2007.

Fuente: Elaboración propia sobre la base de Mineduc 2006 y SIES (2007 - 2015).

La permanencia de estudiantes matriculados baja dramáticamente al séptimo año, exhibiéndose una mayor permanencia en las universidades del CRUCH y privadas, probablemente por la extensión de las carreras.

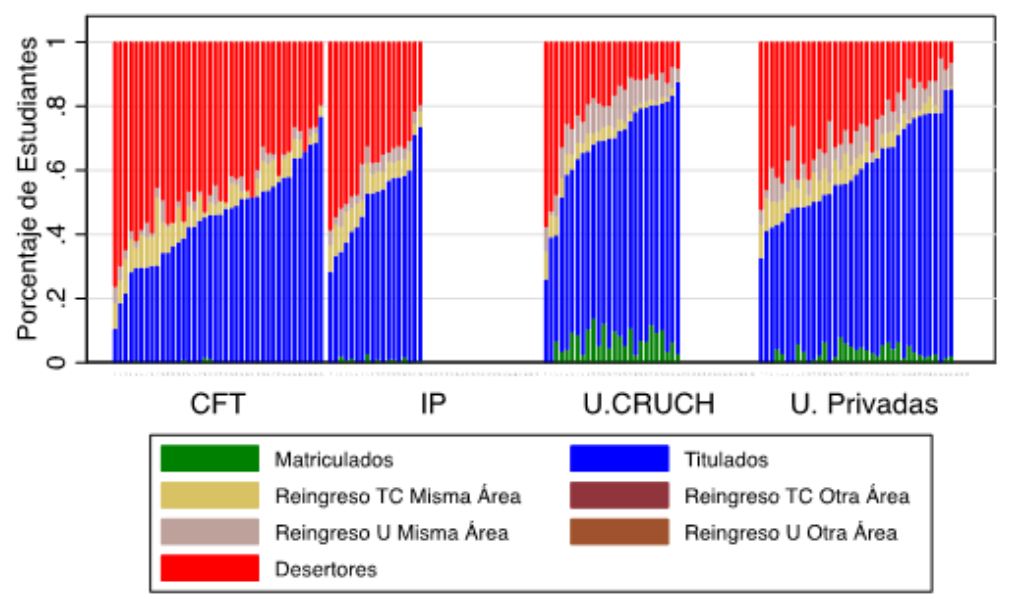

Figura 1D. Porcentaje de estudiantes por tipo de institución y por año según condición de matrícula, titulación, deserción y reingreso de los graduados de educación secundaria en 2006 que ingresaron a la educación superior en 2007.

Fuente: Elaboración propia sobre la base de Mineduc 2006 y SIES (2007 - 2015). 
Incluso en el noveno año se mantiene un porcentaje de estudiantes matriculados en las universidades. Para todas las instituciones, los titulados van predominando, con grandes diferencias entre ellas de todos modos. Llaman la atención dos instituciones técnicas que exhiben tasas de titulación mayores a las de su segmento institucional e incluso, mayores a las de varias universidades.

\section{Resultados de regresiones multinomiales}

Tabla $1 \mathrm{M}$

Resultados de regresiones de primera etapa: deserción, matrícula y titulación para estudiantes técnico profesionales

\begin{tabular}{lcc}
\hline & Matriculado & Titulado \\
\hline Variables & Marginal Effects & Marginal Effects \\
\hline Ranking & $0,370^{* * *}$ & $1,049^{* * *}$ \\
Escolaridad madre & $(0,0642)$ & $(0,0690)$ \\
& $0,0211^{* * *}$ & $-0,0175^{* * *}$ \\
Ingreso familiar USD & $(0,00529)$ & $(0,00536)$ \\
& $0,000285^{* * *}$ & $-9,25 \mathrm{e}-05^{*}$ \\
Puntaje PSU & $(3,76 \mathrm{e}-05)$ & $(4,87 \mathrm{e}-05)$ \\
& $0,00479 * * *$ & $0,00328^{* * *}$ \\
Femenino & $(0,000234)$ & $(0,000245)$ \\
& $-0,0486$ & $0,569^{* * *}$ \\
Ayuda financiera & $(0,0330)$ & $(0,0360)$ \\
& $0,331^{* * *}$ & $0,679 * * *$ \\
Modalidad TP & $(0,0364)$ & $(0,0377)$ \\
Constante & $-0,121^{* * *}$ & $-0,225^{* * *}$ \\
& $(0,0384)$ & $(0,0398)$ \\
Standard errors in parentheses & $-3,376^{* * *}$ & $-3,070^{* * *}$ \\
\hline
\end{tabular}

Fuente: Elaboración propia sobre la base de Mineduc 2006 y SIES (2007 - 2015). 
Tabla $1 \mathrm{~N}$

Resultados de regresiones de primera etapa: reingreso para estudiantes universitarios

\begin{tabular}{lll}
\hline & \multicolumn{2}{l}{ Matriculado universitario Titulado } \\
\hline Variable & Marginal effects & Marginal effects \\
\hline Ranking & $1,105^{* * *}$ & $0,928^{* * *}$ \\
& $(0,0393)$ & $(0,132)$ \\
Escolaridad madre & $-0,00178$ & $-0,0436^{* * *}$ \\
\multirow{2}{*}{ Ingreso familiar USD } & $(0,00358)$ & $(0,0108)$ \\
& $9,59 \mathrm{e}-05^{* * *}$ & $-0,000246^{* * *}$ \\
Puntaje PSU & $(1,73 \mathrm{e}-05)$ & $(6,71 \mathrm{e}-05)$ \\
& $0,00468 * *$ & $-0,00356^{* * *}$ \\
Femenino & $(0,000150)$ & $(0,000448)$ \\
& $0,287^{* * *}$ & $0,240 * * *$ \\
Ayuda financiera & $(0,0200)$ & $(0,0674)$ \\
& $0,250^{* * *}$ & 0,0584 \\
Modalidad TP & $(0,0243)$ & $(0,0817)$ \\
Constante & $-0,184^{* * *}$ & $0,271^{* * *}$ \\
& $(0,0315)$ & $(0,0824)$ \\
Standard errors in parentheses & $-2,782^{* * *}$ & $-0,941^{* * *}$ \\
& $(0,0790)$ & $(0,229)$ \\
\hline
\end{tabular}

Fuente: Elaboración propia sobre la base de Mineduc 2006 y SIES (2007 - 2015).

La tabla detalla los resultados del gráfico considerando como variable base para la comparación la condición de deserción, respecto de matriculados y titulados. 